\title{
Preliminary Study On The Data Mining of Prescriptions Containing Schisandra And The Mechanism of Diabetes
}

\section{Yang Su}

Heilongjiang University of Chinese Medicine

Jing Li

Heilongjiang University of Chinese Medicine https://orcid.org/0000-0001-9620-0426

Fan Zhang

Heilongjiang University of Chinese Medicine

Wen Qin

Heilongjiang University of Chinese Medicine

Lun Wu ( $\nabla 35228778 @ q q . c o m$ )

Heilongjiang University of Chinese Medicine

Yue Gao

Heilongjiang University of Chinese Medicine

Qiuhong Wang

Guangdong Pharmaceutical University

Haixue Kuang

Heilongjiang University of Chinese Medicine

Genhong Cheng

University of California

\section{Research}

Keywords: medicine, drugs, network

Posted Date: November 24th, 2021

DOI: https://doi.org/10.21203/rs.3.rs-1081452/v1

License: (c) (i) This work is licensed under a Creative Commons Attribution 4.0 International License.

Read Full License 


\title{
Preliminary Study on the Data Mining of Prescriptions Containing Schisandra and the Mechanism of Diabetes
}

\author{
Yang $\mathrm{Su}^{1}$, Jing $\mathrm{Li}^{1}$, Fan Zhang ${ }^{1}$, Wen $\mathrm{Qin}^{1}$, Lun $\mathrm{Wu}^{2 *}$, Yue $\mathrm{Gao}^{2}$, Qiuhong \\ Wang $^{3}$, Haixue Kuang ${ }^{1 *}$, Genhong Cheng ${ }^{4 *}$ \\ ${ }^{1}$ College of Pharmacy, Heilongjiang University of Chinese Medicine, Harbin 150040, China \\ ${ }^{2}$ Research Institute of Traditional Chinese Medicine, Heilongjiang University of Chinese Medicine, \\ Harbin 150040, China \\ ${ }^{3}$ School of Traditional Chinese Medicine, Guangdong Pharmaceutical University, Guangzhou \\ 510000, China \\ ${ }^{4}$ Faculty of Microbiology and Immunogenetics, University of California, Los Angeles, CA 90095, \\ USA
}

Correspondence: 335228778@.qq.com; 1539930743@.qq.com; dongabeng@sina.com

\begin{abstract}

\section{Background}

Data mining builds a bridge between ancient books of traditional Chinese medicine and modern medicine. It provides a new perspective for the precise compatibility of prescriptions and the scheme of diagnosis and treatment. Meanwhile, the mechanism of schisandrae chinensis and its compound in treating diabetes and diabetic complications is not clear. This limits the clinical application of Schisandra chinensis.
\end{abstract}

\section{Methods}

Apply TCMISS to mine the core drugs with the highest frequency of combined use with Schisandra chinensis to form a new prescription. And analyze the compatibility law. Use databases such as TCMSP, TCMID, PubChem, Swiss arget prediction, disgenet and Cytoscape software. The interaction between drugs and diseases and related pathways were analyzed. In addition, autodock Vina and other software are used to calculate the molecular docking and binding energy between compounds and key targets. Finally, the therapeutic effect of the new prescription of Fructus schisandrae chinensis S.(Core) on diabetic mice was evaluated by biological experiments. 


\section{Results}

The study employed a data mining S.(Core) was obtained. Using network pharmacology method, mainly containing target prediction, network construction, functional enrichment analysis, and molecular docking to systematically research the mechanisms of S (Core) in treating diabetes. The putative targets of S.(Core) that treat diabetes mainly involved AKT1, GAPDH, IL-6, MAPK3, VEGFA and other targets. The functional enrichment analysis suggested that the produced therapeutic effect of S (Core) against diabetes is mediated by synergistical regulation of several biological pathways, AGE-RAGE signaling pathway in diabetic complications , Insulin resistance, HIF-1 and Insulin signaling pathway. The results of molecular docking showed that the core chemical components of S · core had good affinity with the key targets of diabetes. In addition, We confirmed that $\mathrm{S}$. Core can improve glucose and lipid metabolism in diabetic mice. Enhanced islet $\beta$ Quantity and quality of cells. Reduce the inflammatory response in the body. Liver protection.

\section{Conclusion}

This study explored the mechanism of action of S.(Core) in diabetes by multi-component and multi-target multi pathways, which could provide a theoretical basis for the development and clinical application of $\mathrm{S} \cdot($ Core). 


\section{Background}

Diabetes is a serious global public health problem. About $5.6 \%$ of the world population. The prevalence of diabetes in adults in China is $10.9 \%$, and the number of patients in the world is the highest ${ }^{[1]}$. Persistent hyperglycemia and long-term metabolic disturbances can lead to diabetic nephropathy, diabetic ophthalmopathy and nervous system damage. It has a serious impact on the quality of life and overall life span ${ }^{[2]}$. The main drugs for treating diabetes include guanidine, sulfonylurea, benzoic acid derivatives, and so on. $\alpha$ - Glycosidase inhibitors, etc. The main purpose of these drugs is to control blood sugar. It needs to be taken for a long time. With the development of the disease, the dose increases gradually, which will lead to liver and kidney function injury and a variety of complications ${ }^{[3]}$. Traditional Chinese medicine regards diabetes as a "Xiaoke", so the idea of treatment is similar to that of diabetes therapy. Traditional Chinese medicine takes "both symptoms and treatment" as the principle. Through syndrome differentiation and treatment, it can not only effectively reduce blood glucose, but also effectively control complications ${ }^{[4]}$. It has certain advantages in the understanding and treatment of diabetes.

Many herbs have been used in the treatment of diabetes and the development of new drugs. Schisandra chinensis is one of them. Modern pharmacological studies show that the chemical components of Schisandra chinensis mainly include lignans, triterpenes, polysaccharides, volatile oils, flavonoids, organic acids and so on. Schisandra chinensis has many activities, such as protecting liver, anti-oxidation, anti-tumor, anti-aging, enhancing immune function and so $\mathrm{on}^{[5]}$. The extract of Schisandra chinensis has hypoglycemic effect on STZ induced diabetic rats, with little toxic side effects. ${ }^{[6]}$ 。Fructus Schisandra chinensis polysaccharide can significantly reduce fasting blood glucose level, increase fasting serum insulin level and insulin sensitivity index in diabetic rats ${ }^{[7]}$. Schisandra chinensis was not a key herbal medicine for the treatment of "Xiaoke" in ancient times. Schisandra chinensis in treating diabetes mellitus. The Evidence can be found in ancient books and modern basic clinical research. For example, sour Yuxiao Decoction (Schisandra chinensis as the king drug) with good clinical efficacy, Shenggan Jiangtang formula (Schisandra chinensis as the king drug $)^{[8][9]}$, etc. Prescription is the essence of Chinese medicine, which embodies the theoretical characteristics of Chinese medicine "differentiation of symptoms and signs, monarch and discipline", "Jun-Chen-Zuo-Shi", "strengthening the body and eliminating 
evil". Prescription has the characteristics of multi-component, multi-channel and multi-target synergy, and has unique advantages in the prevention and treatment of complex diseases.

However, the mechanism of Schisandrae chinensis and its compound in the treatment of diabetes and diabetic complications is not clear. This limits the clinical application of Schisandra chinensis. This study is based on the Chinese Pharmacopoeia, the online database of yaozhi.com and the tested prescriptions in ancient medical books. TCMISS was used to make frequency statistics and composition law analysis of target prescriptions. Based on the biomolecular network, the correlation mechanism between prescription and disease syndrome was established by using the method of network pharmacology. Analyzing the hidden patterns or laws between traditional Chinese medicine and body. To explore the potential mechanism of Schisandra chinensis seed core drug in treating diabetes mellitus. Using molecular docking and animal in vivo experiments are the key ways to verify the results of network pharmacology. To provide theoretical basis for the compatibility of Fructus schisandrae chinensis prescription and the clinical practice of diabetes prescription. Figure 1 shows the flow chart of this whole analysis.

\section{Methods}

\section{Prescription data mining analysis and standardization}

Collection of traditional Chinese medicine prescriptions for the the treatment of "Xiaoke" or "Diabetes" with Schisandra chinensis in the Chinese Pharmacopoeia of (2020 Edition) ${ }^{[10]}$. Subject to the explicit inclusion of Schisandra chinensis in the prescription; Collect the prescription database of proprietary Chinese medicine provided by Yaozhi online database(https://www.yaozh.com/), which is clearly marked as treatment of thirst or diabetes mellitus. Chinese patent medicine varieties clearly marked for the treatment of "Xiaoke" or "Diabetes" and containing Schisandra chinensis and its processed products (Schisandra by vinegar, Schisandra by wine or Schisandra by honey); Collect 25 ancient medical books such as "Zhouhoubeijifang", "Waitai", "Yitong", "Shengji master record", "Qianjin" and "Danxi master record", Screening "main treatment" or "function" column in the above source prescription; It contains It clearly contains the syndrome names of "Xiaoke", "Shanxiao", "Shangxiao", "Zhongxiao", "Xiaxiao", "Pidan", "Liangxiao", "Xiaodan"; and The prescription composition is 
clear, and the Schisandra chinensis and its processed products shall prevail (The above names are the names of classical $b$ diseases and syndromes of traditional Chinese medicine). Finally, 67 prescriptions containing Schisandra chinensis and its processed products were included.

To ensure the correctness of data mining results, The drug names of the collected prescriptions shall be standardized according to the Chinese Pharmacopoeia of (2020 Edition). Refer to the online database of Chinese Pharmacopoeia (http://www.zhongyaocai360.com/zhonghuabencao/) and Chinese materia medica in 2020 and the national planning textbook for the Tenth Five Year Plan of general higher education of traditional Chinese medicine.

After standardizing the prescription name, input the data information into TCMISS software. TCMISS can carry out data mining on the medication law of clinical prescriptions. The high-frequency drugs, the nature and taste of the drugs in the formula, the frequency of meridian attribution and the drug combination obtained by association rules were analyzed.

\section{Screening of core drug components and targets and disease targets}

In TCMSP database (http://tcmspw.com/tcmsp.php) Schisandra chinensis, Ginseng, Astragalus mongholicus, Rehmannia glutinosa, Ophiopogon japonicus and snakegourd root. If not included in this database, TCMID data set shall be used (http://183.129.215.33/tcmid/)Search. And Combined with literature reports, the components with relevant pharmacological effects were supplemented as potential active components. The SDF structure of the above components was obtained using the PubChem database. Import Swiss target prediction database(http://www.swisstargetprediction.ch/), take the target with prediction score greater than 0 as the drug target. Use OMIM(https://omim.org/), Disgenet(https://www.disgenet.org/), Genecards (https://www.genecards.org/) databases to search with "diabetes" as the keyword. Obtaining disease targets. Mapping the component target with diabetic target and obtaining potential target for diabetes treatment with Schisandra chinensis core drug group.

\section{Construction and analysis of component target network}

Using Cytoscape 3.7.2 software. Construct the network diagram of "drug component disease target". The network analyzer function was used to analyze the main active components of the core drug group (Schisandra chinensis, Ginseng, Astragalus mongholicus, Rehmannia glutinosa, Ophiopogon japonicus and snakegourd root). 


\section{PPI network construction and core target analysis}

The above 145 common targets were input into the String database (https://string-db.org/) for retrieva. Set the protein type as "Homo sapiens". The minimum interaction threshold is 0.4.Obtain the network relationship data of target interaction. It was imported into Cytoscape software to draw the network diagram of protein-protein interaction. The size, color, and depth of the node represent the size of the degree value.

\section{Construction of potential target tissue distribution map}

The target tissue distribution map was constructed through GeneCards database. Inductive target distribution of tissues and organs. In other words, the core drug group treats the tissues and organs with high distribution of diabetes. Reveal potential target sites.

\section{Go enrichment and KEGG pathway analysis}

Using Bioconductor bioinformatics software package based on $\mathrm{R}$ language. The functional enrichment of key target genes go and KEGG was analyzed with $\mathrm{P}$ value $<0.05$ and $\mathrm{Q}$ value $<0.05$. And in Microsoft Excel 2019 form to establish Chinese medicine, ingredients, target and the corresponding relationship between the first 20 diabetes related pathways. Use Cytoscape 3.7.1 software for visual display.

\section{Molecular docking}

Autodock Vina software was used to rank the top five target proteins in the degree of core target. Molecular docking verification with potential active components in the core drug group. Metformin, a traditional therapeutic drug, was compared with glibenclamide, which is widely used in clinic. The 2D structures of traditional Chinese medicine components, metformin and glibenclamide were downloaded from PubMed(https://www.ncbi.nlm.nih.gov/pubmed) database. 3D structures of small molecules were generated using Chem3D software. Download the 3D structure of the core target protein through the PDB(https://www.rcsb.org/) database. The pretreatment of ligand and protein receptor molecules was completed by PyMOL and autodocktools-1.5.6 software. Set the pocket parameters and parameter text file of protein receptor. Batch docking under Linux virtual environment using autodockvina software. The docking results of the five target proteins with the strongest binding activity were displayed by PyMOL software.

Preparation of decoction of Schisandra chinensis core herbal medicine group $(\mathrm{S} \cdot$ Core) 
Take 10g of Schisandra chinensis, Ginseng, Astragalus membranaceus, Ophiopogon japonicus, Rehmannia glutinosa and Snakegourd root respectively. After crushing, add 8 times the amount of water, decoct for 3 times, $2 \mathrm{~h} /$ time, combine the filtrate and concentrate it to $0.5 \mathrm{~g} \cdot \mathrm{ml}^{-1}$ (equivalent to 8 times the human dose), and refrigerate for standby.

\section{Diabetic mice induced by high-fat diet combined with STZ}

In short, six week old male C57BL/6J mice were purchased from Liaoning Changsheng Biotechnology Co., Ltd. (Liaoning, China).The use license number of the experimental unit is SYXK(black) 2018-007. Mice are kept in ventilated cages. Temperature control is $23^{\circ} \mathrm{C}-25^{\circ} \mathrm{C}$. Adapt to a 12 hour light/dark cycle. And free access to water and food. After 1 weeks of adjustment, all mice were randomly divided into control group (8 rats) and diabetic model group (24 rats). The mice in the control group were fed standard diet(D12450B, Xiaoshu Youtai Biotechnology Co., Ltd., Beijing, China). Diabetic mice were fed a high-fat diet continuously(D12492, Xiaoshu Youtai Biotechnology Co., Ltd., Beijing, China). After feeding for 8 weeks, A single dose of STZ $(65 \mathrm{mg} / \mathrm{kg})$ was injected into the diabetic group by intraperitoneal injection (I.P) within 15 minutes (STZ was allocated to fresh $0.05 \mathrm{M}$ citric acid buffer (pH4.5). Continuous injection for three days. The mice in the control group were given the same amount of citric acid buffer. During injection, STZ solution was kept in ice bath and dark environment. 7 days after STZ injection, all mice showed high fasting blood glucose level $(\geq 11.1 \mathrm{mmol} / \mathrm{L})$ and were considered as successful models. The mice were randomly divided into three groups $(8$ in each group): diabetes group (Model), Schisandra core compatibility group (S- Core) and metformin hydrochloride group (MET). S· core $600 \mathrm{mg} / \mathrm{kg}$ and MET300 $\mathrm{mg} / \mathrm{kg}$ were administered orally. The control group and the model group were given the same amount of distilled water.In the last 8 weeks of the experiment, normal saline and drugs were given by gavage. FBG and body weight were measured weekly after administration. Measure food and water intake every day. After 8 weeks of treatment, mice were euthanized and tissues were collected for further analysis.

\section{Biochemical analysis}

Fasting blood glucose (FBG), oral glucose tolerance test (OGTT), intraperitoneal insulin tolerance test (IPITT), standard blood glucose meter and blood glucose test paper (sinocare Inc. Changsha, China). According to the manufacturer's instructions. Serum total cholesterol (TC, A111-1-1), triglyceride (TG, A110-1-1), low density lipoprotein cholesterol (LDL-C, A111-1-1), high density 
lipoprotein cholesterol (HDL-C, A112-1-1), alanine aminotransferase (ALT, C009-2-1), aspartate aminotransferase (AST, C010-2-1) and Serum fasting insulin (fins, ZC-38920), glycosylated hemoglobin (HbA1c, ZC-38711;), tumor necrosis factor and TNF- $\alpha$ were measured by enzyme-linked immunosorbent assay (ELISA, ZCI BIO, Shanghai, China) (LBP, zc-39024), interleukin 6 (IL6, ZC-37988), interleukin 2 (IL-2, ZC-37976); ELISA plates were analyzed by enzyme labeling instrument (IMark, BioRad) at $450 \mathrm{~nm}$ wavelength. Steady state model evaluation - insulin resistance (HOMA-IR). Calculated as: fasting insulin (mul-1) $\times$ Fasting blood glucose $($ mul-1)/22.5.

\section{Tissue sampling and histological staining}

Mice were euthanized with pentobarbital sodium injection $(100 \mathrm{mg} / \mathrm{kg})$. At the same time, inhale ether to maintain anesthesia after fasting for 12 hours. Blood samples were collected from retroorbital hemorrhage after anesthesia. Then centrifuged at $3500 \mathrm{~g}$ for $15 \mathrm{~min}$ to obtain serum for serological detection. The liver and pancreas tissues of different groups were fixed in $10 \%$ neutral buffered formalin for paraffin section. Cut the tissue into 4 pieces $\mu \mathrm{M}$ thick. Stained with hematoxylin and eosin $(\mathrm{H} \& \mathrm{E})^{[11]}$. The quantitative data of histological staining were analyzed by Image Proplus software.

Insulin and glucagon were detected by immunofluorescence staining. Islet specimens were fixed overnight in $4 \%$ PFA $\left(4{ }^{\circ} \mathrm{C}\right)$ and embedded in paraffin blocks. Paraffin sections (3um) or frozen sections (8um) were washed with PBS for 3 times. 15 minutes at a time. $0.2 \%-0.5 \%$ Triton X-100 penetration. 5\% normal Donkey Serum blocking. Primary antibody staining overnight. Fluorescence for primary antibody. Secondary antibody test (Jackson Lab). Sections were stained with DAPI (4',6-diamino-2-phenylindole). Fluorescent fixing medium (Dako). For alloantibodies, opaltm 4-color manual IHC Kit (PerkinElmer) was used for detection. Finally, the above results were semi quantitatively analyzed by image Proplus software.

\section{Western blot analysis}

The liver tissue of mice was extracted with Ripa lysate. Liver tissues were harvested and stored at $-80^{\circ} \mathrm{C}$ for western blot analyses. The cells were lysed with biyuntian Western IP lysate to extract total protein. The total protein of each cell lysate was quantified by BCA protein quantitative Kit (all gold). Draw standard curve. Calculate the concentration of protein sample to be determined. Adjust the sample concentration to be consistent. The primary antibodies used in the experiment 
are as follows: InsR(1: 1000 dilution, ab137747, abcam), P-InsR(1: 1000 dilution, ab60946, abcam), GSK-3ß(1: 500 dilution, ab227208, abcam), P-GSK-3ß(1; 500 dilution, ab75745, abcam), GAPDH primary antibody(1: 1000 dilution, D190090-0100, Sangon Bio), secondary antibody Sangon Bio. Ect chemical developer (GE health, rpn2232) was used to detect and capture protein bands. The data obtained by Western blotting were analyzed by image Proplus software.

\section{Statistical analyses}

The data were represented by means \pm standard deviation (SD). And statistical analyses were performed using one-way analysis of variance (GraphPad Software, San Diego, CA, USA). Results were considered to be statistically significant at $\mathrm{p}<0.05$ between various groups.

\section{Results}

\section{Medication frequency statistics}

Screening of 67 prescriptions "Xiaoke" and "diabetes". The frequency of 130 traditional Chinese medicines included was counted. There are 17 kinds of drugs with frequency $\geq 10$, in Table 1. 130 traditional Chinese medicines are classified according to traditional Chinese medicine edited by Gao Xuemin, in Table 2. It indicates that compatibility with Fructus schisandrae chinensis in treating diabetes is often combined with "tonifying drugs", "clearing away heat drugs" and "collecting astringent drugs" (frequency $>99$ times). These three categories of traditional Chinese medicine are reasonably matched with Schisandra chinensis. The effect of Fructus schisandrae chinensis on diabetes is doubled.

\section{Analysis "medicine nature flavor" and "meridian tropisof"}

Medicine nature. The performance of TCM Medicine naturerefers to the material basis of drug efficacy and the role reflected in the treatment process, in which the characteristics of four Qi and five flavors are an important part. four Qi and five flavors can reflect the properties of the drug itself, suggest the main therapeutic function of the drug, reflect the principles and methods of treatment, and are the basis of clinical prescription. The distribution of traditional Chinese medicine on "Four Qi" are cold(37.94\%), warm(36.1\%), flat (19.66\%), slightly cold(4.76\%), heat $(1.5 \%)$ is shown in Figure 2A. The distribution of traditional Chinese medicine on "five 
flavors" are sweet taste(50.66\%), bitter taste(24\%), sour taste(8.82\%), pungent taste(10.72\%), salty taste(2.94\%), astringent taste(2.85\%) is shown in Figure 2B. Most of the traditional chinese medicine combined with Schisandra chinensis in the treatment of diabetes mellitus is cold, warm and flat. Cumulative frequency is $93.7 \%$.The top three medicine flavor frequencies were sweet taste, bitter taste, pungent taste. The cumulative frequency is $85.38 \%$. In traditional Chinese medicine, the pathogenesis of diabetes is Yin and fluid, the exuberance of dryness-heat. It is worth noting that Yin defi-ciency is the initiating factor, the root cause. Dryness-heat in superficiality. The two are cause and effect other. According to the theory of traditional Chinese Medicine "The more yin deficiency and dryness, the more dryness and heat, and the more yin deficiency". Take cold medicine as the main medication principle. Traditional Chinese medicine believes that "water-wetne" hinders the transportation of "Qi"'water-wetne" generates "phlegm turbidity".So diabetes is induced. "water-wetne" should be resisted with warm medicine. Strengthening clinical curative effect with flat medicine. From the perspective of traditional Chinese medicine to see the pathogenesis of diabetes: "lung and stomach heat, kidney yin deficiency". The clinical treatment is mainly traditional Chinese medicine with sweet-nourishing, bitter-discharge,pungent-warm. In the meantime, The application of bitter cold traditional Chinese medicine should pay attention to whether the spleen and stomach are damaged. Prevent injury to human immunity. Therefore, the Chinese herbal medicine with many characteristics of compatibility is good.

Meridian tropisof. The theory of channel tropism is an important part of the theory of TCM, which can reflect the attribution of TCM and prefers certain viscera, meridians, or specific parts. Among the 67 prescriptions selected for the treatment of "diabetes", the meridian tropisof was as follows: lung(20\%), kidney(18\%), heart(18\%), spleen(14\%), stomach(13\%), liver(10\%), large intestine(3\%), bladder(2\%), gallbladder(2\%), small intestine(1\%) is shown in Figure $2 \mathrm{C}$. The drugs with medication frequency $\geqq 10$ belong to lung meridian included (Schisandra chinensis, Ginseng, Astragalus mongholicus, snakegourd root,Licorice,Anemarrhena,Yam);Drugs for regulating kidney meridian (chisandra chinensis, Rehmannia glutinosa, Poria cocos, Anemarrhena, Chinese wolfberry, Yam, Raw Rehmannia glutinosa, Peony bark, Alisma orientalis);Drugs for regulating heart Meridian(Schisandra chinensis, Ginseng, Ophiopogon japonicus, Rehmannia glutinosa, Licorice, Poria cocos, Coptis chinensis, Chinses angelica, Tree peony bark);Drugs for regulating spleen meridian(Ginseng, Ophiopogon japonicus, Astragalus 
membranaceus, Licorice, Poria cocos, Pueraria lobata, Coptis chinensi, Yam, Chinses angelica);Drugs for regulating stomach meridian(Ophiopogon japonicus, Snakegourd root, Licorice, Pueraria lobata, Anemarrhena asphodeloides, Coptis chinensi). Lung governing management and regulation.Lung also plays an indispensable role in body fluid metabolism the main source of water. The traditional Chinese medicine for returning the spleen, stomach and heart meridian has the functions of nourishing kidney yin, warming and recuperating kidney yang, tonifying the heart yin. It shows that the Chinese herb of Schisandra chinensis has compatibility and general tendency in treating diabetes mellitus.

High frequency core drug group. Association rule Apriori algorithm was used to analyze the rule of prescriptions containing schisandra chinensis. According to the number of screening prescriptions and the number of traditional Chinese medicine used.

Combined with experience judgment and different parameters to extract data preread. Set "support" to 10, 20, 30, and "confidence" to 0.9 . According to the above conditions, data mining and analysis were carried out for core drug combinations. It turns out that when "support" is set to 10. It can comprehensively show the use of drugs compatible with schisandra chinensis. As the "support level" increases to 30 , the core drug compatibility component becomes prominent. Schisandra chinensis is often used in combination with Ginseng, Ophiopogon japonicus, Rehmannia glutinosa, Astragalus mongholicus and Snakegourd root in the treatment of diabetes. Network display under different "support" is shown in Figure 2D. In the famous medical books of ancient books, all these traditional Chinese medicines have prominent therapeutic effects on diabetes. Therefore, the above 6 closely related traditional Chinese medicines are recombined. As a drug combination with schisandra chinensis as the core S.(Core). In order to explore the mechanism of their treatment of "diabetes" and carry out follow-up studies.

\section{Screening of core drug components and obtaining results of action targets}

Set $\mathrm{OB} \geqslant 30 \%$ and DL $\geqslant 0.18$ in TCMSP database. The effective components of Schisandra chinensis, Astragalus mongholicus, Ginseng and snakegourd root were screened. Searching Rehmannia glutinosa and Ophiopogon japonicus in TCMID database. The schisandrin B and schisandrin a included in Schisandra chinensis were supplemented combined with the literature;

Ginsenoside Rg1, Ginsenoside Rg3, ginsenoside Rg5, ginsenoside Rb1, ginsenoside RB3, ginsenoside $\mathrm{Re}$ and ginsenoside $\mathrm{RD}$ in ginseng;Astragaloside $\mathrm{IV}$ in Astragalus 
membranaceus ${ }^{[12],[13],[14],[15],[16],[17],[18],[19],[20],[21]}$. Additional file 1: Table S1 displayed the elaborated data of active components of S.(Core). A total of 139 potential active components were obtained. 975 drug targets were screened using Swiss Target Prediction database.

The keywords "diabetes" were searched in OMIM, Disgenet and Genecards databases. A total of 1136 disease targets were obtained after removing the rrepeat. 975 drug targets and 1136 disease targets were input into Venny2.1 online software mapping tool platform. A Venn diagram was drawn, and 145 drug-disease common targets were obtained after the intersection of the two (Figure 3A). Details about S.(Core)-genes, diabetes-genes, and S.(Core)-disease overlapping target genes were presented in Additional file 2: Table S2.

\section{Construction of component target network}

The 139 potential active components of the core drug group and 145 drug-disease common targets were input into Cytoscape software. Delete the isolated components that have no intersection with the target. The network of drug-component-target-disease interactions was plotted (Figure 3B). Network graph topology analysis is performed using Network Analyzer.Degree value indicates the number of correlation between the component and the target. The higher the Degree value is, the more critical the component is in the network ${ }^{[22]}$. Additional file 3:Table S3. The TCM components in additional file 3 all involve multiple targets. May have a wide range of pharmacological activities. It has certain curative effect on diseases of heart, liver, spleen, lung, kidney and nervous system. It can predict the effects of various TCM components in the core drug group containing Schisandra chinensis on specific targets of biological network. Comprehensive synergistic effect on the treatment of diabetes.

\section{PPI network analysis and core target screening}

To further explore the relationship between potential targets at protein level. The 145 common targets above are entered into the String database for retrieval. The conditional protein species was Homo sapiens and the minimum interaction threshold was 0.4.Obtain network relationship data of target interaction. The above results were imported into Cytoscape software to draw the protein interaction network diagram (Figure 3C). The size, color, and shade of the node represent the size of the Degree value. These targets may be highly regulatory in the treatment of diabetes.

\section{Target tissue distribution}

Cytoscape 3.7.1 software was used to construct the potential target-tissue distribution network 
map of the core drug group containing Schisandra chinensis. Reflect the degree of interaction between target and organization (Figure 3D). 168 nodes and 2173 edges are obtained. The circular node represents the potential target. Arrow nodes represent tissues and organs with target distribution. The darker the color of the arrow, the higher the distribution proportion of the target in the tissue and organ. The results showed that 145 potential targets were highly expressed in nervous system (140), blood (136), liver(133), muscle (133) respectively. Additional file 4: Table S4 Targets and Tissues Organs.

\section{Enrichment results and analysis of GO and KEGG pathways}

GO function enrichment analysis was performed on 145 common targets in R language.Items were sorted according to $\mathrm{P}$ size (threshold $\mathrm{P}<0.05$ ), and biological process $(\mathrm{BP})$, cell component (CC) and molecular function (MF) were selected, as shown in Figure 4A-C. The potential targets were enriched to 155 KEGG signaling pathways. The first 20 paths are shown in a bar chart, as shown in Figure 4D. The results showed that the potential key network targets in the core drug group were significantly enriched in diabetes complications, insulin resistance, inflammation, cancer, lipid metabolism, cell cycle and other related pathways. Among them, the disease-related pathways are most involved. It is suggested that the core drug components of Fructus schisandrae chinensis can regulate glucose metabolism through insulin resistance, AGE-RAGE, HIF-1, insulin and other signaling pathways, so as to achieve the effect of treating diabetes.Details about results of Gene Ontology (GO) and KEGG pathways were presented in Additional file 5.

\section{The core compounds in $S \cdot($ Core) are docked with key proteins}

The top five key targets of degree in PPI network were used to conduct preliminary verification of molecular docking. Target protein receptors for VEGFA (PDF ID 6V7K), AKT1 (PDF ID 3096), GAPDH (PDF ID 3GPD), IL6 (PDF ID 409H), TNF(PDF ID 2ZPX) were downloaded from RCSB PDB database. Verification with TCM ingredients with degree $\geqslant 14$ (Table 3). Listed metformin and glyburide were selected as positive drugs for docking with the target (Table 4). It is generally believed,Binding energy $<0$ has binding activity, $<-5$ has better binding activity, $<-7$ has strong binding activity ${ }^{[23]}$. The results show that the minimum binding energy of all molecules to the target protein is less than 0 . Suggesting that ligand and receptor can spontaneously bind. Moreover, The docking results with the strongest binding activity of the five target proteins are that the same component oleanolic acid comes from Ophiopogon japonicus. The docking model is 
shown (Figure 5).

\section{In vivo experimental validation}

Effects of S.(Core) on blood glucose, insulin resistance and pancreas function in diabetic mice. Network pharmacology results indicate that diabetes is associated with increased IR and impaired glucose tolerance. Therefore, we studied the effects of S.(Core) on blood glucose control, insulin sensitivity and pancreas protection in diabetic mice. A sixteenth week OGTT analysis (Figure 6E) and fasting blood glucose (Figure 6A) showed that high fat diet successfully induced diabetes. As shown in figures $6 \mathrm{~A}$ and $\mathrm{B}$, Compared with the model group, $\mathrm{S} \cdot(\mathrm{Core})$ group significantly reduced fasting hyperglycemia and hyperinsulinemia $(\mathrm{p}<0.05)$. The results showed that $\mathrm{S} \cdot(\mathrm{Core})$ group improved blood glucose and insulin homeostasis in diabetic mice. Meanwhile, compared with the model group, the HOMA-IR index of the (S·Core) group was lower (Figure 6C) $(\mathrm{P}<0.05)$. Administration of $\mathrm{S} \cdot($ Core) and metformin could effectively reduce the level of $\mathrm{HbAlc}(\mathrm{P}<0.05)$ (Figure 6D). It shows that S.(Core) group has a long-term effect on alleviating hyperglycemia. OGTT and ipitt analysis at the end of the test showed that, S.(Core) treatment increased glucose tolerance in diabetic mice effectively (Figure $6 \mathrm{E})(\mathrm{P}<0.05)$. And increased insulin sensitivity (Figure 6F) $(\mathrm{P}<0.05)$. Compared with the blank control group, the islets in the model group shrank and the morphology was destroyed. Compared with the model group, the islet morphology in the $\mathrm{S} \cdot($ Core) group was significantly restored and the degree of atrophy was better reversed (Figure $6 \mathrm{G})(\mathrm{P}<0.05)$. It indicated that $\mathrm{S} \cdot(\mathrm{Core})$ components could significantly improve pancreatic injury and protect pancreatic tissue in diabetic mice. In addition, immunofluorescence experiments showed that, Pancreatic islets in diabetic mice $\beta$ the number of cells decreased significantly. but $\alpha$ the number of cells increased significantly and the distribution was more uniform(Figure $6 \mathrm{H})(\mathrm{P}<0.05)$. Metformin or $\mathrm{S} \cdot(\mathrm{Core})$ treatment not only increased $\beta$ the number of cells decreased $\alpha$ number of cells. This shows a good protective effect on pancreatic islets in diabetic mice. All these analyses confirmed the anti diabetic effect of S.(Core). It is characterized by regulating blood glucose, insulin homeostasis and insulin sensitivity.

Effects of S.(Core) on blood lipids, liver and inflammation in diabetic mice. H\&E dyeing and visual shooting display. Compared with the control group, the liver of the model group showed inflammation and abnormal lipid accumulation. However, S·(Core) improved significantly after treatment (Figure 7A) $(\mathrm{P}<0.05)$. We also detected two important transaminases ALT and AST. The 
results also support the above conclusions (Figure 7F,G) $(\mathrm{P}<0.05)$. Hyperlipidemia is a major risk factor for diabetes mellitus. It is characterized by increased lipid accumulation in serum ${ }^{[24]}$. Compared with the blank group, the model group significantly increased the levels of TC and TG in serum . After 16 weeks of treatment, it was found that the levels of serum TG and TC in the $\mathrm{S}$ (Core) group were significantly lower than those in the model group. The curative effect was similar to that of metformin group (Figure $7 \mathrm{~B}, \mathrm{C})(\mathrm{p}<0.05)$. Interestingly, compared with the model group, the serum LDL-C level in the S.(Core) group decreased and the HDL-C level increased (Figure 7D,E) $(\mathrm{p}<0.05)$.

In addition, according to the pathway enrichment analysis results. Based on the pathway enrichment analysis, targts(TNF- $\alpha$, IL-6, IL-2, INSR, GSK-3 $\beta$ ) from two of the most significant pathways, AGE-RAGE signaling pathway in diabetic complications, and Insulin resistance, were selected for further experimental validation. The results showed that the levels of serum inflammatory factors in diabetic mice increased significantly. After S.(Core) intervention, inflammation was significantly reduced by reducing these inflammatory cytokines (Figure $7 \mathrm{H}, \mathrm{I}, \mathrm{J})(\mathrm{p}<0.05)$. Meanwhile, the systemic inflammatory regulation of $600 \mathrm{mg} / \mathrm{kg} \mathrm{S} \cdot($ Core) treatment group was similar to that of $300 \mathrm{mg} / \mathrm{kg}$ met treatment group $(\mathrm{P}>0.05)$. Western blot showed that the drug treatment significantly blocked the inhibitory effect of high fat diet on INSR phosphorylation $(\mathrm{P}<0.05)$. And GSK-3 $\beta$ the level of phosphorylation increased significantly (Figure $7 \mathrm{~K}, \mathrm{~L})(\mathrm{p}<0.05)$. In conclusion, $\mathrm{S} \cdot($ Core) intervention can reduce serum lipid accumulation. Improve liver protection. Significantly reduce the level of serum inflammatory cytokines. Promote IRS1 and GSK-3 $\beta$ Phosphorylation of.

\section{Discussion}

Diabetes is a metabolic disease caused by various causes. This disease is easy to cause macrovascular, microvascular and nervous system diseases. The incidence rate and disability rate of diabetes and its complications increase year by year. It has become the third major killer that seriously affects human health after tumor and cardiovascular disease. Hypoglycemic drugs are the main treatment for diabetes. On the basis of oral hypoglycemic drugs single treatment, combined treatment or insulin combined with oral hypoglycemic drugs. To control the change of 
blood glucose level throughout the day and delay the progress of the disease, it is necessary to take medicine for life. However, if the medication period is prolonged, the body's drug resistance can gradually increase, resulting in limited drug effect. Chinese medicine is a popular treatment for diabetes in recent years. In a large number of reports, practical therapeutic effects and complication prevention effects were obtained. It is found that Schisandra chinensis has therapeutic effect on diabetes mellitus. However, the lack of clinically effective prescriptions containing Schisandra chinensis in the market limits its clinical application. In order to better summarize and inherit the academic thought and medication experience of doctors. Data mining and network pharmacological analysis were used in this study. We screened and collected anti diabetic Chinese herbal compound containing Fructus schisandrae chinensis. To clarify its molecular mechanism and provide basis for new drug research and development and clinical application. 67 prescriptions met the standard. The "frequency", "efficacy", "meridian tropism", "four Qi" and "five flavors" were analyzed and counted by tcmiss. The compatibility frequency of "Schisandra chinensis - ginseng" is the highest. This combination is a classic clinical compatible drug pair, which originates from Shengmai Powder in Qianjin prescription. The combination of the two drugs can enter the lung and kidney meridians, and has the effects of generating fluid and restoring Qi, nourishing yin and generating fluid, tonifying deficiency and strengthening ${ }^{[25]}$. The drugs used to treat diabetes with Fructus Schisandrae are mainly supplementation and clearing away heat, while astringent drugs are auxiliary. Traditional Chinese medicine believes that the treatment of diabetes should take "deficiency of Qi and Yin" as the root cause, "dryness and heat", "blood stasis" as the standard, "body fluid consumption injury" as the symptom. Therefore, in addition to taking dialectics as the basis, the formulation focuses on strengthening the body's immunity (adding tonic drugs for strengthening the body's health and strengthening the foundation), controlling the replication of the virus (selecting heat clearing and detoxifying drugs), and preventing excessive urine sugar and urine (astringent drugs with the dual effects of solid absorption and fluid generation). In the compatibility of traditional Chinese medicine, Schisandra chinensis has high frequency and strong correlation with ginseng, Astragalus membranaceus, Ophiopogon japonicus, Rehmannia glutinosa and trichosanthin. Combine the six traditional Chinese medicines into a new prescription. Interestingly, we found that the new prescription is similar to the prescription for treating diabetes mellitus (Huang Qitang, Sheng Mai San, Sheng Di 
Huang Yin Zi, Yiqi Yangyin Huoxue Fang, etc.). From the perspective of modern pharmacology, schisandrin $\mathrm{B}^{[26]}$, ginsenoside ${ }^{[27]}$, astragaloside $\mathrm{IV}^{[28]}$, Rehmannia glutinosa ${ }^{[29]}$, Ophiopogon japonicus polysaccharides ${ }^{[30]}$ and total saponins ${ }^{[31]}$, Snakegourd $\operatorname{root}^{[32]}$ can resist diabetes. It can effectively improve the symptoms of diabetes, and meet the needs of modern clinical hypoglycemic treatment.

The development of network pharmacology provides a new idea for the exploration of pharmacodynamic components of traditional Chinese medicine. The five traditional Chinese medicines mined in this paper have the strongest correlation with Schisandra chinensis, and are set as new prescriptions. Using network pharmacology research methods. Explore the mechanism of new prescription in treating diabetes mellitus from microcosmic perspective. The PPI network analysis showed that the core targets of the new prescription containing Schisandrae chinensis against diabetes were AKT1, VEGFA, GAPDH, IL6 and TNF. AKT1, also known as protein kinase B, is mainly involved in the regulation and release of cytokines, apoptosis and glucose metabolism. The expression of AKT1 protein was down regulated and PI3K/Akt signaling pathway was activated, so as to improve the glucose decomposition ability of cells ${ }^{[33]}$. The expression of vascular endothelial growth factor (VEGFA) is correlated with diabetes mellitus. VEGFA can lead to inflammatory factor secretion, donation and moistening ${ }^{[34]}$. Hyperglycemia inhibits the key glycolytic enzyme glycerol 3-phosphate dehydrogenase (GAPDH) by over activating poly ADP ribose polymerase (PARP), which causes the accumulation of glycolytic intermediates and finally increases the glucose level ${ }^{[35]}$. The activation of inflammatory response by innate immune system can lead to cell apoptosis, tissue fibrosis and organ dysfunction, resulting in insulin resistance, impaired insulin secretion and renal failure. IL-6 plays a certain role in the inflammatory cascade ${ }^{[36]}$. In 1993, an article in science first introduced the relationship between tumor necrosis factor (TNF) and obesity, and the relationship between inflammation and insulin resistance ${ }^{[37]}$. TNF can affect insulin sensitivity and islets $\beta$ Cell function through the action of blood and/or paracrine, which in turn causes IR. We selected the common drugs on the market (metformin and glibenclamide) as the positive control of molecular docking. The results of molecular docking showed that the binding energies of important active compounds and main targets were less than $-2 \mathrm{~kJ} / \mathrm{mol}$, indicating that they had good affinity and binding activity. In conclusion, the target may protect islets by down regulating the activity of inflammatory factors 
and inhibiting insulin resistance $\beta$ Cells maintain blood glucose homeostasis, thereby improving the symptoms of diabetes.

BP analysis showed that the core drug group was involved in monosaccharide metabolism, glucose metabolism and blood glucose homeostasis. The regulation of blood glucose homeostasis depends on the synergy of multiple tissues and organs. Each tissue senses or regulates blood glucose by absorbing, consuming and storing glucose or releasing hormones and metabolites. For diabetic patients, severe fluctuations in blood sugar can cause damage to human organs. And accelerate the occurrence and development of Diabetic Acute and chronic complications. Therefore, maintaining blood glucose homeostasis is very important. KEGG enrichment analysis showed that the core drug group involved AGE-RAGE signaling pathway, insulin resistance pathway and HIF-1 signaling pathway in diabetic complications. AGEs can act directly on islets $\beta$ Cells, induced islets $\beta$ Cells respond to oxidative stress, Cause insulin synthesis and secretion dysfunction, promote islets $\beta$ cells Apoptosis ${ }^{[38]}$. $\mathrm{ZHU}^{[39]}$ et al found that AGEs interacts with RAGE, triggering a series of inflammatory reactions, leading to diabetes and its complications. IR is an important part of diabetes. It has been reported that over $70 \%$ of diabetic patients have IR, and IR is also the common pathological basis of hypertension, coronary heart disease, metabolic syndrome, polycystic ovary syndrome and other diseases. HIF- $\alpha$ It is closely related to the occurrence and development of diabetes and its complications ${ }^{[40]}$. Under the condition of high glucose and hypoxia, a large amount of HIF- $\alpha$ will accumulate in cells. Exacerbate the ischemic hypoxia state of diabetic patients and promote the occurrence of diabetic complications ${ }^{[41]}$. These indicate that the development of diabetes and complications is related to insulin resistance, blood glucose homeostasis and immune disorders.

The results of tissue distribution of potential targets in Schisandra chinensis core drug group showed that 145 potential targets were highly expressed in nervous system, blood and liver respectively. Recent studies have shown that the liver is not only an important place for glucose and lipid metabolism, but also one of the main target organs of insulin. Hepatic insulin resistance is the key cause of impaired fasting glucose, and is also an important factor in the occurrence and development of diabetes ${ }^{[42]}$. Traditional Chinese medicine believes that, liver governing free coursing. The liver plays a role in regulating and controlling the dynamic process of blood glucose 
production, distribution, application, storage and metabolism. Liver failing to govern free coursing. The food could not be distributed in time. It turns into sugar turbidity and soaks the blood vessels. The external manifestation is that the postprandial blood glucose is high and fluctuates greatly ${ }^{[43]}$. Compared with continuous hyperglycemia, blood glucose fluctuation can aggravate oxidative stress, inflammatory reaction and accelerate the occurrence of complications ${ }^{[44,45,46]}$. The function of "liver" in the system of traditional Chinese medicine includes some functions of the liver in the anatomical sense. Modern medicine believes that the liver regulates the fluctuation of blood glucose through the synthesis and decomposition of liver glycogen and gluconeogenesis. When the liver is damaged, it directly affects normal glucose metabolism and produces impaired glucose tolerance. And diabetes and microvascular complications. This is similar to "liver failing to government free coursing" in traditional Chinese medicine. At the same time, the characteristics of multi-target tissue distribution reflect the integrity of the body. It indicates that the core drug group of Schisandra chinensis has the potential to treat diabetes combined with other diseases.

Finally, according to the above analysis, we confirmed the effectiveness of S.(Core) and verified the key pathway through in vivo animal experiments. The pancreas and liver were evaluated pharmacologically. We found that on the one hand, S.(Core) can reduce FBG, HbA1c, OGTT, ITT, fins and HOMA-IR and protect islets $\beta$ The role of cells. On the other hand, S·(Core) can repair liver damage in diabetic mice, reduce the levels of TC, TG, LDL-C, AST and ALT in serum of diabetic mice, and significantly increase the content of HDL-C. It shows that S.(Core) has a good effect on improving lipid metabolism disorder and protecting liver in diabetic mice. In addition, we selected blood and liver for verification according to the distribution of potential target tissues. AGEs-RAGE can activate NF-kB. NF-kB activated by AGEs-RAGE signaling pathway enters the nucleus, which can cause a large number of adhesion molecules, growth factors and pro-inflammatory cytokines (such as TNF- $\alpha$, IL-6, IL-2). Therefore, it causes tissue injury and inflammatory reaction, which is an important mechanism of T2DM and its complications $^{[47]}$. INSR on hepatocyte surface would activate insulin receptor substrate (IRS) after binding with insulin, and then functionally mediate downstream like glucose/lipid metabolism, hepatocyte proliferation, DNA synthesis, and cell cycle regulation ${ }^{[48]}$. GSK-3 is a post insulin receptor signal protein with two isomers GSK-3 $\alpha$ And GSK-3 $\beta$. It belongs to constitutive activated kinase, that is the phosphate state of GSK-3 is inactivated and the dephosphorylation state is 
activated. Normally, insulin phosphorylates GSK-3 $\beta$ Inactivate it, inactivated GSK-3 $\beta$ It loses the inhibitory effect on hepatic glycogen synthesis and can promote hepatic glycogen synthesis. In type 2 diabetes, insulin resistance occurs in the body. Absolute or relative deficiency of insulin efficacy. Dephosphorylated GSK-3 $\beta$ increase. the synthesis of liver glycogen decreases and the content of liver glycogen decreases, resulting in the increase of blood glucose level ${ }^{[49]}$. The regulation of S.(Core) on the key targets of these two pathways was confirmed by serum biochemistry and Western blotting.

\section{Conclusion}

To sum up, this study summarized the law of compatibility and common drug combinations of Fructus schisandrae chinensis in the treatment of diabetes through data mining. Using different databases to explore the rule of traditional Chinese medicine in the treatment of diabetes. Research strategy of pharmacology based on Network. Integrate various database information and use molecular docking for preliminary verification. To elucidate the mechanism of the new prescription of Fructus schisandrae chinensis in treating diabetes with multiple components and multiple targets. The reliability of network pharmacological prediction was verified by animal experiments in vivo. However, traditional Chinese medicine compound is not a simple chemical aggregate. The human body is an organic whole. Data mining, network pharmacology, molecular docking analysis and animal experiments are limited to theoretical verification. Further experimental and clinical verification of the above potential pharmacodynamic components, action targets and action mechanism is also needed. Because this study only investigates the effectiveness of the new prescription. Therefore, the same dose is used for each traditional Chinese medicine in the prescription. In the future, we will study and discuss the formula meaning analysis, dose dependence and toxicity of Schisandra chinensis new prescription. 


\section{Abbreviations}

TCM: Traditional Chinese Medicine; STZ: Streptozocin; TCMISS: Traditional Chinese Medicine Inheritance Support Platform; PPI: Protein-protein integration; TCMSP: Traditional Chinese medicine system pharmacology analysis platform; TCMID: Traditional Chinese Medicine Integrated Database; OMIM: Online Mendelian Inheritance in Man; GO: Gene ontology; KEGG: Kyoto Encyclopedia of Genes and Genomes; S·core: Schisandra chinensis core herbal medicine group; FBG: Fasting blood glucose; OB: Oral bioavailability; DL: Drug-likeness; IR: insulin resistance;

\section{Declaration}

\section{Ethics approval and consent to participate}

The animal experiment complies with the publication of guidelines for the care and use of laboratory animals of the National Institutes of Health (NIH Publication No. 80231, revised in 978). It was approved by the experimental animal Committee of Heilongjiang University of traditional Chinese medicine.

\section{Consent for publication}

Not applicable.

\section{Availability of data and materials}

The datasets used and analyzed during the current study are available from the corresponding author on reasonable request.

\section{Competing interests}

The authors declare that they have no competing interests.

\section{Funding}

This work was jointly supported by grants from the University Nursing Program for Young Scholars with Creative Talents in Heilongjiang Province (Grant No.UNPYSCT-2016207), Research Project of Harbin Science and Technology Innovation Talents (Grant No. 2017RAQXJ214)(RC2017QN003037), the Selective Financial Aid for Returnees from Overseas Studies in Heilongjiang Province (Start-up Category) (Grant No. 2018QD0011)(2018383), Outstanding Innovative Talents Project of Heilongjiang University of Traditional Chinese Medicine-Young Academic Leaders Project (Grant No.2018RCD12)(201877), Post doctoral research start up project in Heilongjiang Province-LBH-Q19183), the Outstanding youth of project by Natural Science Foundation of Heilongjiang Province of China (YQ2020H030), The work was also supported by the Outstanding youth of project by Natural Science Foundation of Heilongjiang Province of China (Grant No.YQ2019H028). Traditional Chinese Medicine Inheritance and Innovation the "Hundred-Thousand-Ten Thousand Project" Talent Project-Chief Scientist of Qihuang Project, Traditional Chinese Medicine Inheritance and Innovation the

"Hundred-Thousand-Ten Thousand Project” Talent Project (Qihuang Project) - QiHuang Scholar.

\section{Authors' contributions}

YS and K-HX conceived and designed the study. C-GH and W-QH drafted the manuscript. LJ and ZF collected the data. WL, GY and QW performed the data analysis. R-WC provided advice 
during the study and manuscript preparation. All authors read and approved the final manuscript.

\section{Acknowledgements}

Not applicable.

\section{References}

[1] Saeedi P, Petersohn I, Salpea P, Malanda B, Karuranga S, Unwin N, Colagiuri S, Guariguata L, Motala AA, Ogurtsova K, Shaw JE, Bright D, Williams R. Global and regional diabetes prevalence estimates for 2019 and projections for 2030 and 2045: Results from the International Diabetes Federation Diabetes Atlas. Diabetes Res Clin Pract. 2019;157:107843.

[2] Lan XB, Zhou G, Sun YH. Progress in pathogenesis and drug therapy of diabetic complications. Chin J of Clinical Rational Drug Use. 2021;14(05):178-181.

[3] Hou JY, Wan P, Zhang YQ, Tang SH, Liu F, Yu LP, Xu HY. Discussion on Prescription

Discovery and Molecular Mechanism of Portulacae Herba in Treating Diabetes Mellitus Based on Data Mining and Integrative Pharmacology. Chinese Journal of Experimental Traditional Medical Formulae. 2020;26(24):1-10.

[4] Yu BR, Liu HF, Du Q, Nan Q, Guo Y, Early diagnosis of type 2 diabetes with "new disease entering the collaterals". Journal of Traditional Chinese Medicine. 2020;61(03):209-212.

[5]Ma YC, Feng TT, Han YB, Fan C C, Liu YF, Liang S, Wang XQ, Dai LJ. Advances in chemical constituents andpharmacology of Schisandra chinensis. Acta Chinese Medicine and Pharmacology.2020;48(11):67-71.

[6] Yang JH, Sun CB, Geng JN, Li QJ, Zhu R, Chen XX, Chen AT, Yu XY. Effect of extract of Schisandra chinensis onexpression of matrix metalloproteinase in Kidney tissue of diabetic rats and its protective effect on kidney tissue. Journal of Jilin University (Medicine Edition). 2017;43(03):512-517+667.

[7] Deng RS, Zhu XQ. Effects of Schisandra Polysaccharide on the Expressions of Rho A/ROCK Signaling Pathway Protein in Colon Muscular Layer of T2DM Rats. Western Journal of Traditional Chinese Medicine. 2020;33(10):25-29.

[8] Zhu ZD, Gu LM. Treatment of 60 cases of type II diabetes with sour and sweet promoting method. Liaoning Journal of Traditional Chinese Medicine. 1998;(01):25.

[9] Gao YB, Wang Y, Feng XZ. Summary of the first National Conference on diabetes in traditional Chinese Medicine. Journal of Traditional Chinese Medicine. 1991;(05):52-55.

[10] National Pharmacopoeia Committee. Chinese Pharmacopoeia 2020 Edition (Volume I) [M]. Beijing: China Pharmaceutical Science and Technology Press, (2020).

[11] Jiang H, Tong Y, Yan D, Jia S, Ostenson CG, Chen Z. The soybean peptide vglycin preserves the diabetic $\beta$-cells through improvement of proliferation and inhibition of apoptosis. Sci. Rep. 2015;5(1):1-16.

[12] Mou ZX, Feng ZG, Xu Z, Zhuang F, Zheng XY, Li XK, Qian JC, Liang G. Schisandrin B alleviates diabetic nephropathy through suppressing excessive inflammation and oxidative stress. Biochem Biophys Res Commun. 2019;508(1):243-249.

[13] Yi D, Qian C, Wang GM, Yan PS, Liang SZ, Wang J. Wang, Schizandrin A Protects Human Retinal Pigment Epithelial Cell Line ARPE-19 against HG-Induced Cell Injury by Regulation of miR-145. Mol Ther Nucleic Acids. 2020;19:42-49.

[14] Wang T, Gao YB, Yue RC, Wang XL, Shi YM, Xu JY, Wu BJ, Li YM. Ginsenoside Rg1 Alleviates Podocyte Injury Induced by Hyperlipidemia via Targeting the mTOR/NF- к B/NLRP3 Axis. Evid Based Complement Alternat Med. 2020;2735714.

[15] Yang RY, Jiang X, He XQ, Liang DL, Sun SS, Zhou GY. Ginsenoside Rb1 Improves Cognitive Impairment Induced by Insulin Resistance through Cdk5/p35-NMDAR-IDE Pathway. Biomed Res Int. 2020;5:(20)3905719.

[16] Xie WJ, Zhou P, Qu MW, Dai ZR, Zhang XL, Zhang CY, Dong X, Sun GB, Sun XB. Ginsenoside Re Attenuates High Glucose-Induced RF/6A Injury via Regulating PI3K/AKT Inhibited HIF-1 a /VEGF Signaling Pathway. Front Pharmacol. 2020;11:695. 
[17] Wei YG, Yang HX, Zhu CH, Deng JJ, Fan DD. Hypoglycemic Effect of Ginsenoside Rg5 Mediated Partly by Modulating Gut Microbiota Dysbiosis in Diabetic db/db Mice. Agric Food Chem. 2020;68(18):5107-5117.

[18] Kaviani M, Keshtka S, Azarpira N, Aghdaei MH, Geramizade h B, Karimi MH, Yaghobi R, Esfandiari E. Shamsaeefar A, Nikeghbalian S, Al-Abdullah IH. Cytoprotective effects of ginsenoside Rd on apoptosis-associated cell death in the isolated human pancreatic islets. EXCLI J. 2019;18:666.

[19] Wang HY, Wu W, Wang GX, Xu WZ, Zhang FQ, Wu B, Tian TP. Protective effect of ginsenoside Rg3 on lung injury in diabetic rats. J Cell Biochem. 2019;120(3):3323-3330.

[20] Meng FL, Su XT, Li W, Zheng YN. Ginsenoside Rb3 strengthens the hypoglycemic effect through AMPK for inhibition of hepatic gluconeogenesis. Exp Ther Med. 2017;13(5):2551-2557.

[21] Leng B, Li C, Sun Y, Zhao K, Zhang L, Lu ML, Wang HX. Protective Effect of Astragaloside IV on High Glucose-Induced Endothelial Dysfunction via Inhibition of P2X7R Dependent P38 MAPK Signaling Pathway. Oxid Med Cell Longev. 2020;5070415.

[22] Pu DQ, Liu Z, Liang JW, Zhou C, Hao QZ. To explore the mechanism of Reduening injection inhibiting inflammatory storm of Novel Coronavirus pneumonia based on network pharmaclogy and molecular docking. Pharmacology and Clinics of Chinese Materia Medica.

2020;36(04):12-17.

[23] Huang Y, Yang SS, Lin X, Zhao SJ, Wei XY, Fu CM, Zhang Z. The mechanism of action of Fuzi-Lizhong pill in treatment of ulcerative colitis based on network pharmacology-molecular docking. Pharmaceutica Sinica. 2020;55(08):1812-1822.

[24] Carr MC, Brunzell JD. Abdominal obesity and dyslipidemia in the metabolic syndrome: importance of type 2 diabetes and familial combined hyperlipidemia in coronary artery disease risk. J Clin Endocrinol Metab. 2004;89(6):2601-7.

[25] Zhang YT, Li SH, Zhang CY. Analysis of the effects of threeTraditional Chinese medicine in shengmayin. China Practical Medicine. 2020;4(23):130-131.

[26] Li L, Wang RF, Zhang LX, Chen L, Shi ZF. Effects of Schisandrin B on Oxidative Stress and Inflammatory Response in Type 2 Diabetic Rats. Chin J Mod Appl Pharm.

2020;37(15):1812-1817.

[27] Xu J, Dong JX, Wang P, Qiu ZD, Yiao F. Research Progress of Ginsenoside Rg3 in Treatment of Obesity- Induced Type 2 Diabetes Mellitus. Chinese Archives of Traditional Chinese Medicine. 2021;13(03):1-12.

[28] Yu CJ, Zhao JD, Yang D, Du X, Wu JP, You LZ, Fang ZH. Preventive and Therapeutic Effects of Astragaloside IV on Retinopathy in Diabetic Rats and Its Effects on Oxidative Stress Pathway. Chinese Archives of Traditional Chinese Medicine. 2020;38(11):194-197+ 292.

[29] Wang L. Effects of Rehmannia glutinosa on intestinal flora in diabetic mice. Peking Union Medical College. 2013.

[30] Song N, Su DF, Liu XY, Gao Y, Liu C, Shan XJ. Effects of Ophiopogon japonicuson polysaccharide on serum SOD,GSH-Px,CAT and MDA in diabetic peri-menopausal rats. J Southeast Univ (Med Sci Edi). 2019;38(06):979-984.

[31] Liu X, Zhang AP, Sun JQ, Chen KL. Study on the hypoglycemic effect of different extracts. Chin J Hosp Pharm. 2009;29(09):719-721.

[32] Yan JX. Preparation and evaluation of trichosanthin polysaccharide/astragalus polysaccharides hydrogel. Lanzhou University of Technology. (2018).

[33] Du HC. Effects of Ranolazine Combined with Xueshuantong Capsule on Cell Autophagy and Myocardial NF- к B, p-Akt, IP7 Levels During Myocardial Ischemia-reperfusion Injury in Diabetic Rats. China Journal of Pharmaceutical Economics. 2020;15(09):39-43.

[34] Hong M, Li QD, Shi RX. Isoliquiritin' s inhibition on the proliferation and migration of high glucose-induced ARPE-19 cells via VEGFA / NF- $\kappa$ B pathway regulation. Chinese Traditional Patent Medicine. 2020;42(10):2616-2621.

[35] Zhu HM, Zhu SL, Li XY, Chen JH, Jiang Y, Ye L. Effect of glucose-control and $\mathrm{N}$-Acetylcysteune on PARP and GAPDH actichronic kidney disease in diabetes. Chin J Diabes. 2013;21(05):426-428.

[36] Mesgari-Abbasi M, Ghaderi S, Khordadmehr M, Nofouzi K, Tayefi-Nasrabadi H, McIntyre G. Enteroprotective effect of Tsukamurella inchonensis on streptozotocin induced type 1 diabetic rats. Turkish Journal of Biochemistry. 2019;44(5):683-691. 
[37] Hotamisligil GS, Shargill NS, Spiegelman BM. Adipose expression of tumor necrosis factor-alpha: direct role in obesity-linked insulin resistance. Science. 1993;259(5091): 87-91. [38] Lim M, Park L, Shin G, Hong H, Kang I, Park Y. Induction of apoptosis of $\beta$ cells of the pancreas by advanced glycation end-products, important mediators of chronic complications of diabetes mellitus. Annals of the New York Academy of Sciences. 2008;1150(1):311-315.

[39] Zhu YX, Shu TT, Lin Y, Wang HD, Yang JW, Shi YG, Han X. Inhibition of the receptor for advanced glycation endproducts (RAGE) protects pancreatic $\beta$-cells. Biochemical and biophysical research communications. 2011;404(1):159-165.

[40] Girgis CM, Cheng K, Scott CH, Gunton JE. Novel links between HIFs, type 2 diabetes, and metabolic syndrome. Trends Endocrinol Metab. 2012;23(8):372-380.

[41] Xia MH, Huang RL, Sun Y, Semenza GL, Aldred SF, Witt KL, Inglese J, Tice RR, Austin CP. ldentification of chemical compounds that induce HIF-1a activity. Toxicol Sci.

2012;112(1):153-163

[42] Pansuria M, Xi H, Li L, Yang XF, Wang H. Insulin resistance, metabolic stress, and atherosclerosis. Front Biosci (Schol Ed). 2012;4:916-31.

[43] Pu LH, Li JJ, Liao L, Zeng M, Wu ML, Yin LP. Discussion on Blood Glucose Fluctuation based on"Liver Controlling Dispersion". Clinical Journal of Traditional Chinese Medicine. 2020; 32(11):2008-2010.

[44] Zoppini g, Verlato G, Targher G, Bonora Enzo, Trombetta M, Muggeo M. Variability of body weight,pulse pressure and glycaemia strongly predict total mortality in elderly type2diabetic patients. The Verona Diabetes Study. Diabetes Metab Res Rev. 2008;24(8):624-628.

[45] Muggeo M, Zoppini G, Bonora E, Brun E, Bonadonna RC, Moghetti P, Verlato G. Fasting piasma glucose variability predicts 10-year surviv of type 2 diabetes patients:The Verona Diabetes Study. Diabetes care. 2000;23,(1):45-50.

[46] Finfer S, Chittock D, Li Y, Foster D, Dhingra V, Bellomo R, Cook D, Dodek P, Hebert P, Henderson W, Heyland D, Higgins A, McArthur C, Mitchell I, Myburgh J, Robinson B, Ronco J. Intensive versus conventional glucose control in critically ill patients with traumatic brain injury: long-term follow-up of a subgroup of patients from the NICE-SUGAR study. Randomized Controlled Trial. 2000;23,(1):45-50.

[47] Yamagishi S. Role of advanced glycation end products (AGEs) and receptor for AGEs (RAGE) in vascular damage in diabetes. Exp Gerontol. 2011;46(4):217-224.

[48] Han L, Yang H, Zheng YJ, Wei XX, Dan WC, Zhang LL, Ding LL, Ding QY, Ma X, Wang XM, Zhao LH, Tong XL. Mechanism exploration of Gouqi-wentang formula against type 2 diabetes mellitus by phytochemistry and network pharmacology-based analysis and biological validation. Chin Med. 2021;16(1):1-18.

[49] Patel S, Doble B, Woodgett JR.Glycogen synthase kinase-3 in insulin and Wnt signalling: a double-edged sword? Biochem Soc Trans. 2004;32(5):803-808. 
Table 1 The drugs with frequency more than 10 in screening prescriptions

\begin{tabular}{ccc|ccc}
\hline NO. & Name of TCM & Frequency & NO. & Name of TCM & Frequency \\
\hline 1 & Schisandra chinensis & 67 & 10 & Anemarrhena & 18 \\
2 & Ginseng & 42 & 11 & Chinese wolfberry & 12 \\
3 & Ophiopogon japonicus & 34 & 12 & Coptis chinensis & 12 \\
4 & Astragalus membranaceus & 32 & 13 & Yam & 11 \\
5 & Rehmannia glutinosa & 32 & 14 & Raw Rehmannia glutinosa & 11 \\
6 & Snakegourd root & 30 & 15 & Chinses angelica & 10 \\
7 & Licorice & 29 & 16 & Tree peony bark & \\
8 & Poria cocos & 22 & 17 & Alisma orientalis
\end{tabular}


Table 2 Analysis of Drug Types in Prescriptions Containing Schisandra for Treating Diabetes

\begin{tabular}{|c|c|c|}
\hline Category & Name of TCM & Frequency \\
\hline \multirow[t]{9}{*}{ Tonifying herbs } & Ophiopogon,japonicus, Astragalus membranaceus,Licorice, Chinese & 215 \\
\hline & wolfberry, Yam,Raw Rehmannia glutinosa, Chinses angelica, Cistanche & \\
\hline & deserticola,Morinda officinalis,Dendrobium,Jade bamboo,Asparagus & \\
\hline & cochinchinensis,Dodder,Pilose antler,Polygonatum,Loquat leaf,Radix & \\
\hline & Paeoniae Alba,Jujube,Eucommia ulmoides,North Sea & \\
\hline & Cucumber,Psoralea corylifolia,Atractylodes & \\
\hline & macrocephala,Codonopsis pilosula,Flatstem Milkvetch & \\
\hline & Seed,Ligustrum lucidum,Acanthopanax senticosus,Honey,Alpiniae & \\
\hline & oxyphyllae Fructus,Radix Pseudostellariae & \\
\hline \multirow[t]{4}{*}{ Heat-clearing herbs } & Rehmannia glutinosa,Snakegourd root,Anemarrhena,Coptis & 138 \\
\hline & chinensis,Peony bark,Pericarp,Plaster,Figwort,Baikal & \\
\hline & Skullcap,Phellodendron amurense,berberidis,Gardenia,Rhizoma & \\
\hline & Picrorhizae & \\
\hline \multirow[t]{3}{*}{ Astringent herbs } & Schisandra chinensis, Cornus officinalis,Dark & 99 \\
\hline & plum,Hematite,Limonitum,Mantis egg-case,Raspberry,Lotus & \\
\hline & seed,Semen Euryales, Gallnut & \\
\hline Inducing diuresis for & Poria cocos,Alisma orientalis,Poria with hostwood,Dioscorea & 41 \\
\hline \multirow[t]{2}{*}{ treating strangurtia herbs } & hypoglauca Palib,Pyrrosia lingua,Tetrapanax papyriferus,Coix & \\
\hline & seed,Corn whisker,Polygonum cuspidatum & \\
\hline Treating exterior & Pueraria lobata,Cinnamon & 35 \\
\hline syndromes herbs & Twig,Ginger,Windbreak,Bupleurum,Perilla frutescens crispa & \\
\hline \multirow[t]{2}{*}{ Qi-regulating herbs } & Fructus aurantii,Litchi seed,The root of three-nerved & 12 \\
\hline & spicebush,Sandalwood,Dried tangerine peel,Woody fragrance & \\
\hline Phlegm-reducing and & Loquat leaf,Trichosanthes, Chinese bellflower,Fructus & 8 \\
\hline antitussive herbs & Trichosanthis,Mulberry bark & \\
\hline Promoting blood & Salvia miltiorrhiza, Rhizome of chuanxiong,Achyranthes & 15 \\
\hline circulation and Removing & bidentata,Peach kernel & \\
\hline
\end{tabular}


blood stasis herbs

Sedative herbs

Interior-warming herbs

Digestive herbs

Anthelmintic herbs

Liver-eliminating

wind-relieving herbs

Purgative sherbs

Resuscitation herbs

Dampness-transforming

herbs

Antirheumatic herbs

Hemostatics herbs

Antipruritic herbs
Magnet,Fossil fragments, Polygala root,Cinnabar

20

Monkshood,Cinnamon,Dried ginger

Gallus gallus domesticus,malt

Pumpkin,Betel nut

Oysters,Bezoar

4

Chinese rhubarb,Sesame Seed

2

Acorus tatarinowii,borneol

2

Agastache rugosus,Cardamom

2

Papaya

1

Rhizoma Imperatae

1

realgar 
Table 3. The target protein and corresponding components of the core drug group containing Schisandra for the treatment of diabetes $(\mathrm{kcal} / \mathrm{mol})$

\begin{tabular}{|c|c|c|c|c|c|}
\hline Traditional Chinese medicine composition & AKT1 & VEGFA & GAPDH & IL6 & TNF \\
\hline oleanolicacid & -10.6 & -13.2 & -14.5 & -12 & -14.3 \\
\hline Spinasterol & -8.7 & -10.6 & -10.9 & -9.4 & -10.6 \\
\hline Celabenzine & -7.8 & -8.8 & -10.2 & -8.5 & -9.8 \\
\hline ophiopogonanone c & -7.6 & -9.5 & -10.3 & -9 & -10.7 \\
\hline methyl ophiopogonanone a & -7.5 & -8.9 & -10.3 & -8.6 & -10.4 \\
\hline Gomisin G & -7.2 & -6.9 & -10.8 & -8.7 & -10.4 \\
\hline methyl ophiopogonanone $b$ & -7.1 & -9.7 & -9.5 & -8.1 & -9.5 \\
\hline orchinol & -7.1 & -8.4 & -8.3 & -7.9 & -8.3 \\
\hline Girinimbin & -7 & -7.5 & -7.9 & -7.4 & -8.3 \\
\hline isorhamnetin & -7 & -8.4 & -9.2 & -8.6 & -9.4 \\
\hline quercetin & -7 & -7.3 & -8.4 & -7.4 & -8.7 \\
\hline Schizandrin A & -6.6 & -8.5 & -8.3 & -7.3 & -8.8 \\
\hline $\begin{array}{l}\text { (6aR,11aR)-9,10-dimethoxy-6a,11a-dihydro-6H-benzofurano[3,2-c]chrome } \\
\text { n-3-ol }\end{array}$ & -6.5 & -6.5 & -7.3 & -6.8 & -7.8 \\
\hline kaempferol & -6.5 & -7.7 & -8 & -7.6 & -8.6 \\
\hline Panaxadiol & -6.5 & -7.5 & -8.6 & -7.4 & -8.2 \\
\hline n-[beta-hydroxy-beta-(4-hydroxyphenyl)]ethyl-4-hydroxy cinnamide & -6.3 & -7.6 & -7.9 & -7.4 & -9 \\
\hline rehmapicrogenin & -6.3 & -6.9 & -7.1 & -6.4 & -8.1 \\
\hline Wuweizisu C & -6.1 & -6.9 & -7.2 & -6.9 & -7.5 \\
\hline Schizandrer B & -6 & -6.1 & -7.2 & -7.7 & -7.4 \\
\hline ferulic acid methyl ester & -5.9 & -6.1 & -6.7 & -6.7 & -7.8 \\
\hline Gomisin-A & -5.9 & -7.6 & -6.5 & -6.3 & -7 \\
\hline Gomisin B & -5.8 & -8.7 & -7.9 & -8.3 & -9.4 \\
\hline
\end{tabular}


(3R)-3-(2-hydroxy-3,4-dimethoxyphenyl)chroman-7-ol

$\begin{array}{lllll}-5.6 & -6.9 & -8 & -6.7 & -6.3 \\ -5.5 & -7.2 & -7.6 & -7.4 & -7.1 \\ -5.4 & -5.9 & -6.3 & -7.1 & -7.6 \\ -5 & -5.5 & -7.3 & -6.2 & -6.7 \\ -4.9 & -5.6 & -6.6 & -6.5 & -6.7 \\ -4.9 & -6.2 & -8.5 & -6.5 & -7.1 \\ -2.7 & -4 & -5 & -4.4 & -5.1\end{array}$

Table 4. The target protein of the core drug group containing Schisandra for the treatment ofdiabetes and the docking of positive drugs $(\mathrm{kcal} / \mathrm{mol})$

\begin{tabular}{lccccc}
\hline Positive drug & AKT1 & VEGFA & GAPDH & IL6 & TNF \\
\hline Metformin & -7.9 & -9.6 & -8.7 & -8.9 & -9.8 \\
Glibenclamide & -4.6 & -5 & -6 & -6.3 & -5.4 \\
\hline
\end{tabular}


Fig.1 Workflow of this study

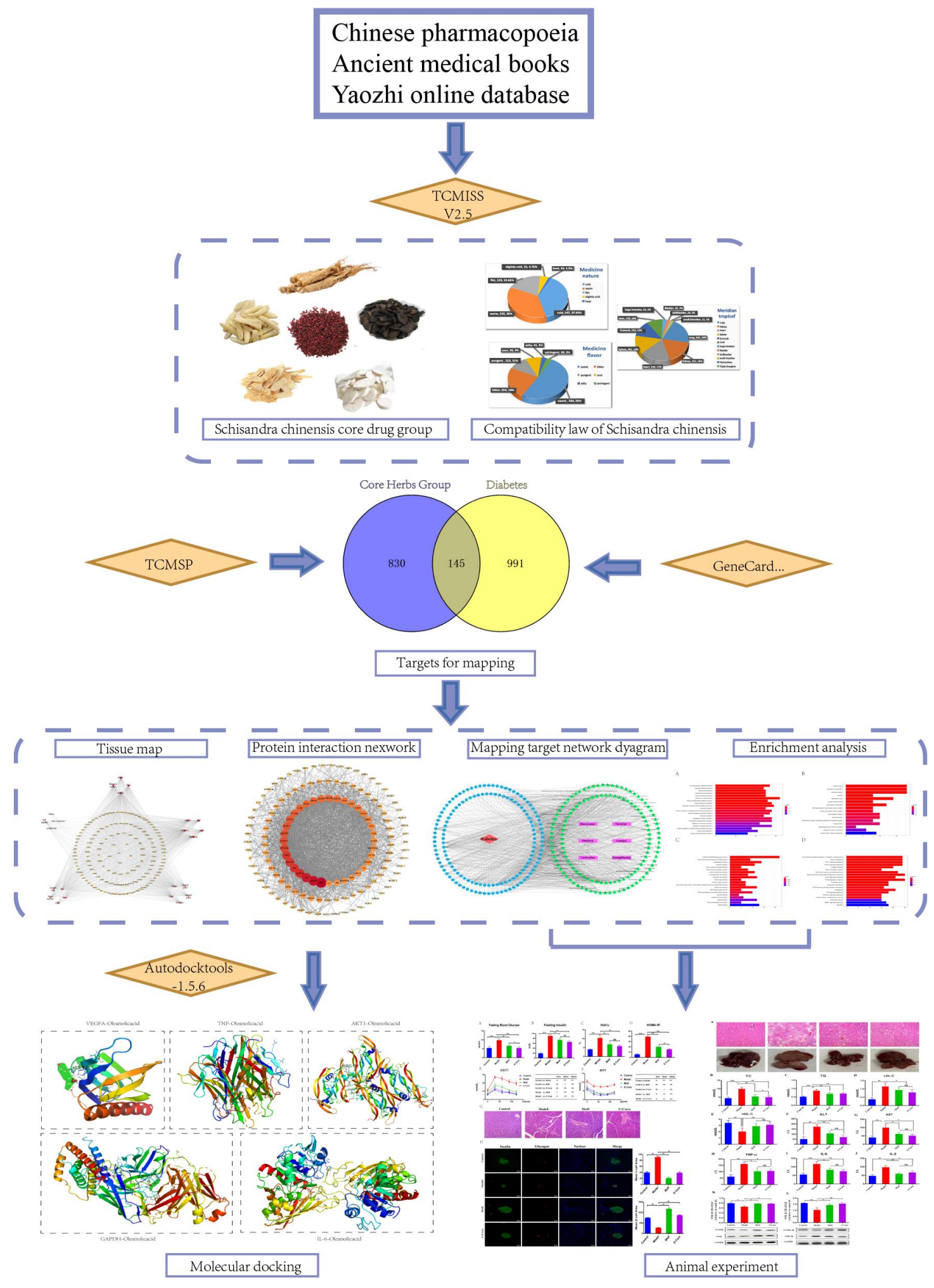


Fig.2 Data mining analysis. A Medicine nature. B Medicine flavor. C Meridian tropisof. D Radar map of drug association rules analysis in screening prescriptions.
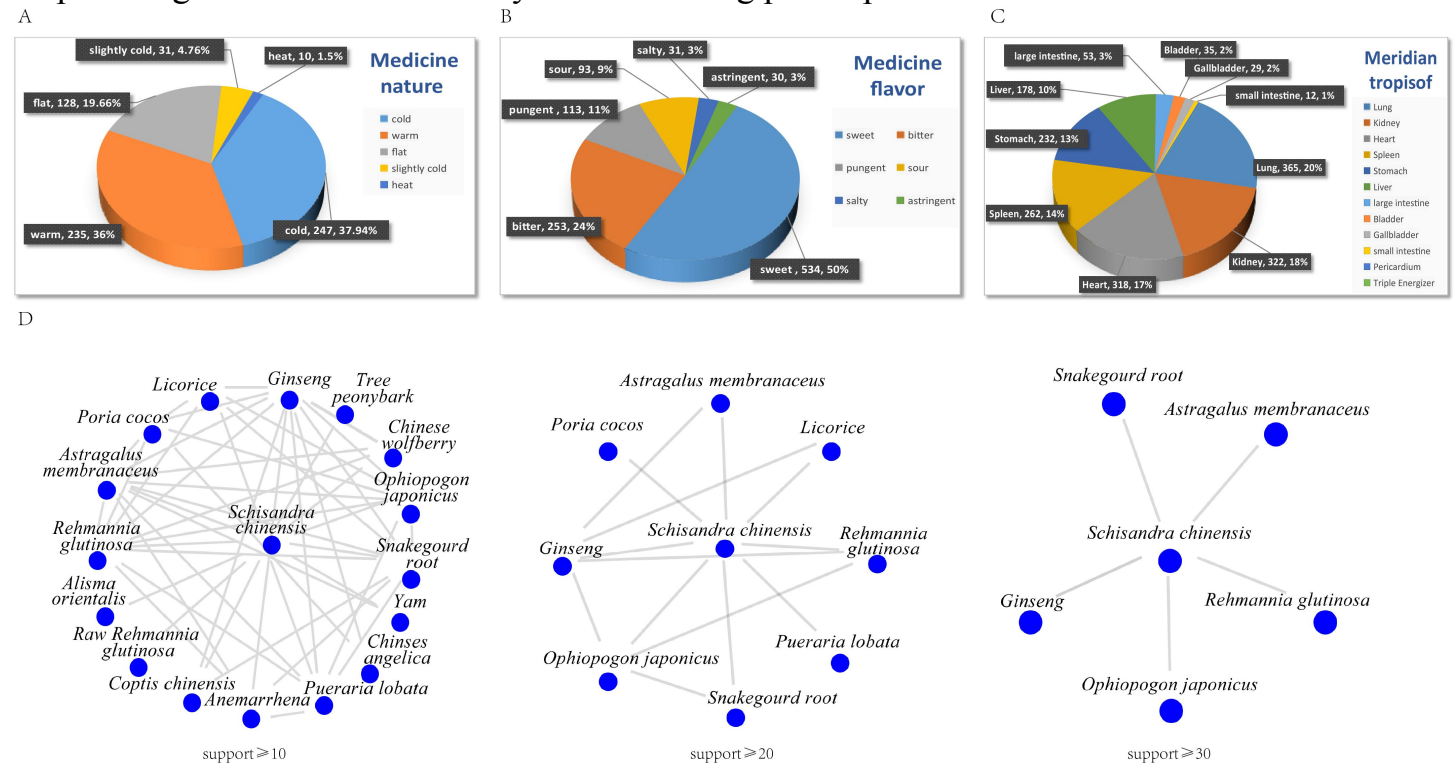
Fig.3 Overlapped targets analysis between "S-core" potential targets and "Diabetes" targets. A Common targets of drug and disease. B Interactions network of drug-component-target-disease . C The PPI network of the key target protein in the treatment of diabetes with the core drug group containing Schisandra. D Target - tissue and organ network diagram.
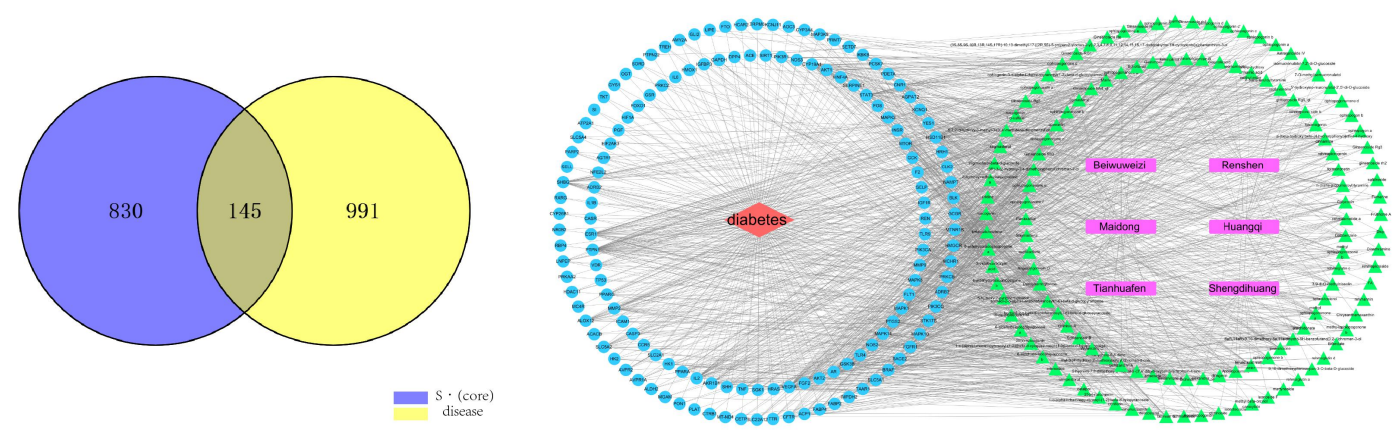

C
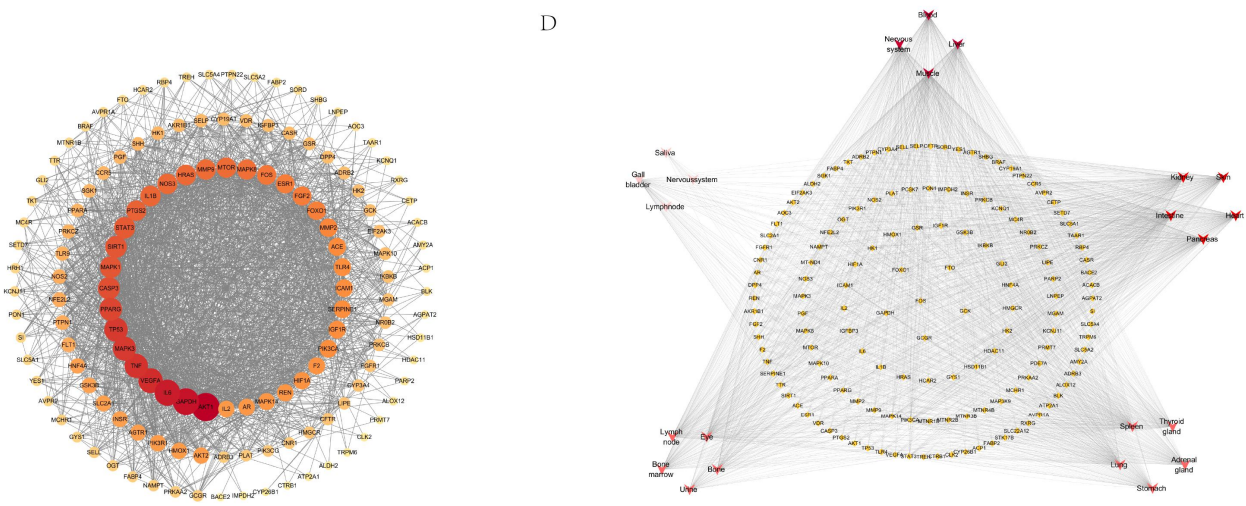
Fig.4 Enrichment analysis of the core targets from PPI analysis. Enrichment analysis of A biological processes $\mathbf{B}$ cell components $\mathbf{C}$ molecular functions. D KEGG signaling pathway analysis in the treatment of diabetes mellitus by the core drug group containing Schisandra chinensis.

A

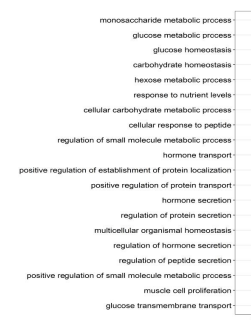

C
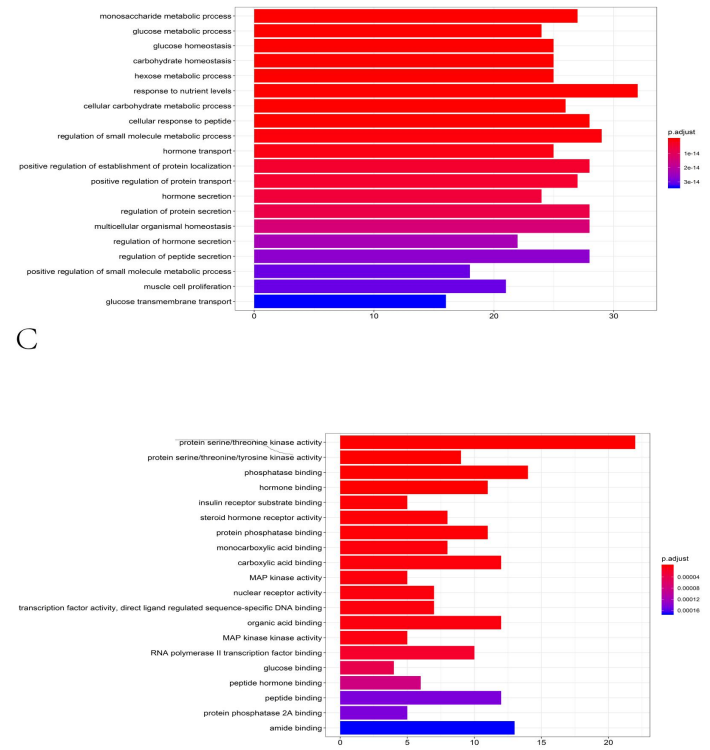

B

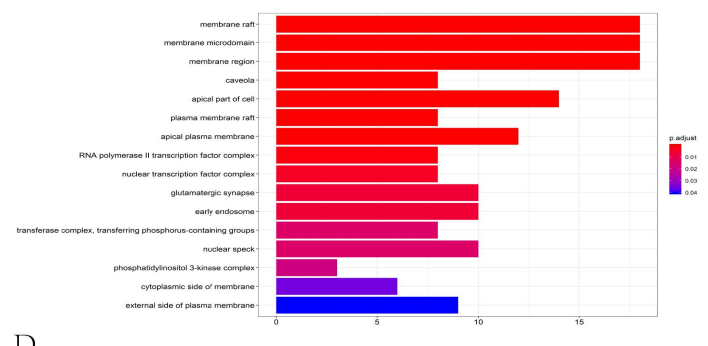

$\mathrm{D}$

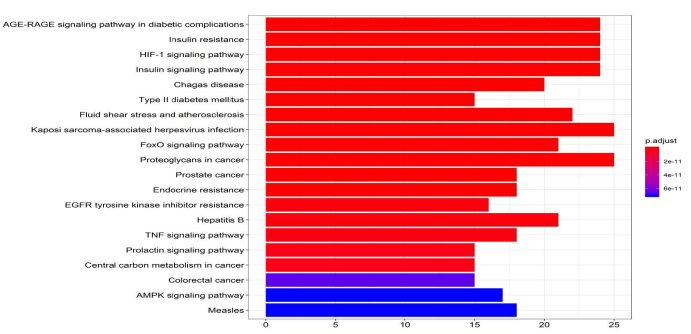


Fig.5 Docking Models of components and core targets of core drug group containing Schisandra in treatment of diabetes.

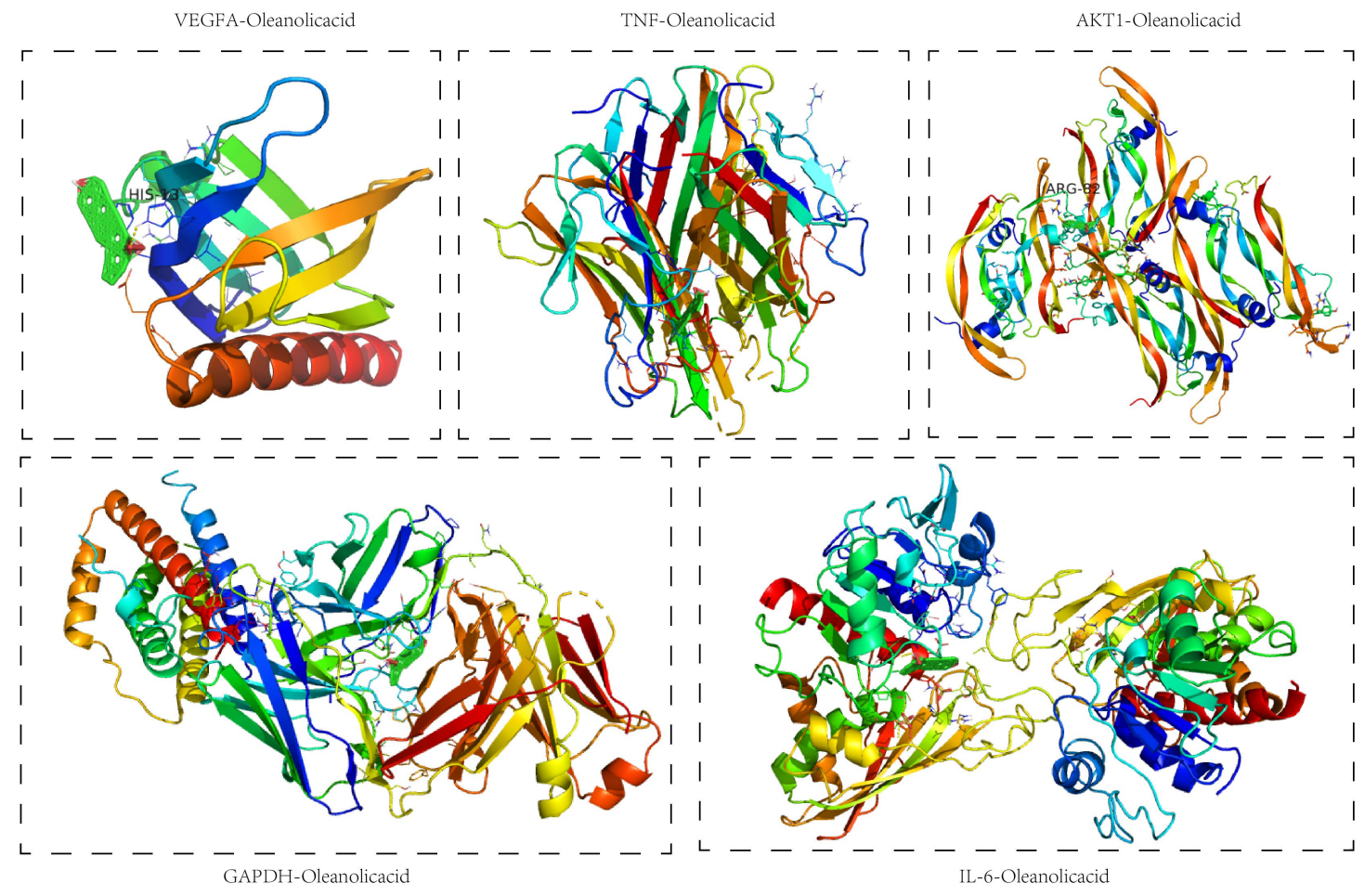


Fig.6 S (Core) administration can improve glucose insulin homeostasis in diabetic mice. A Fasting blood glucose. B fasting insulin. C HbA1c. D HOMA-IR. E OGTT curve at the end of the test. F Ipitt curve at the end of the test. $\mathbf{G}$ Islet H \& E staining. $\mathbf{H}$ Immunofluorescence staining of insulin (green), glucagon (red) and nucleus (blue). Scale: $50 \mu \mathrm{m}$. The data are exprssed as mean

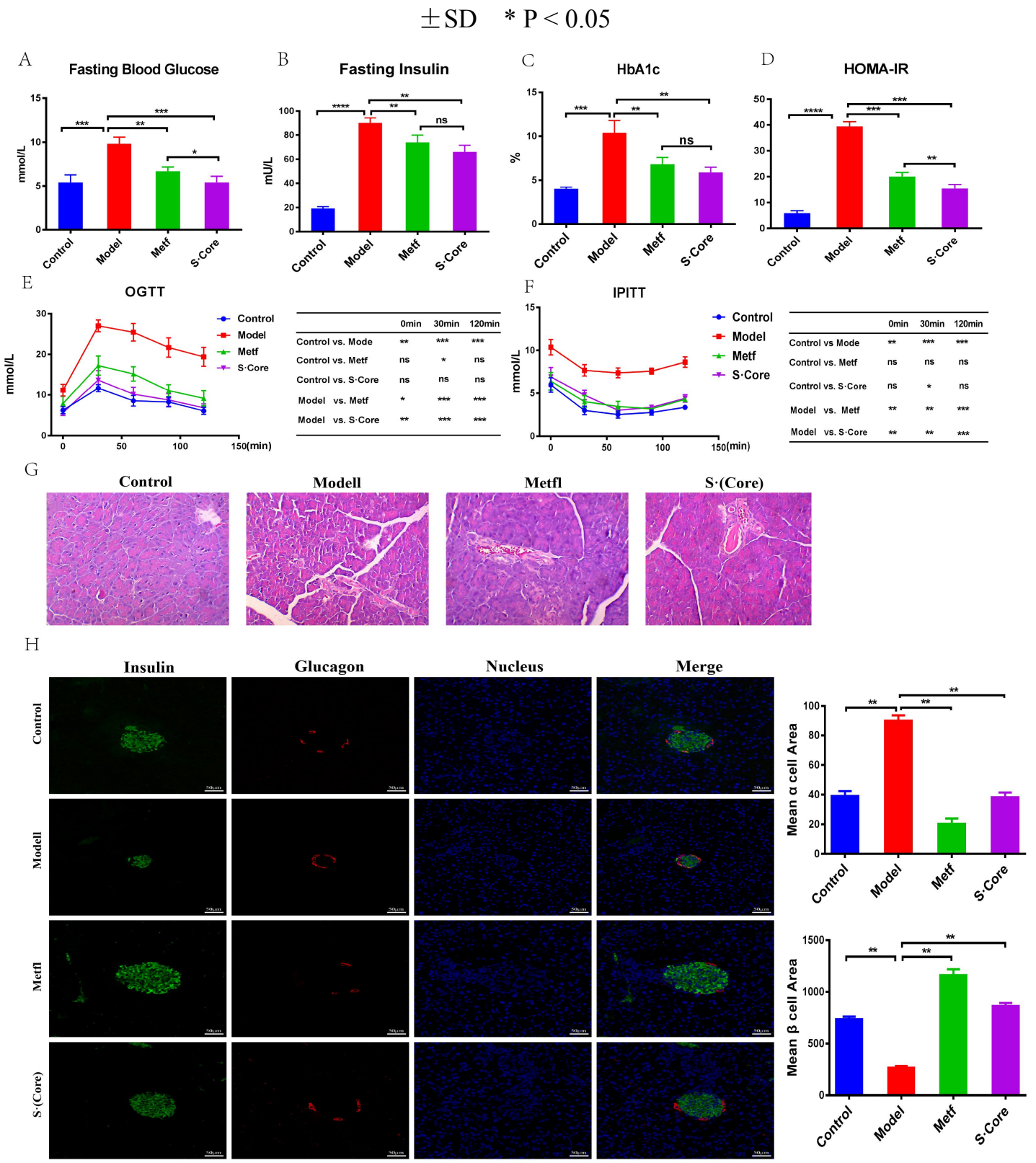


Fig.7 Effects of S.(Core) on blood lipids, liver and inflammation in diabetic mice. A Liver H \& E staining. B Serum level of TC, C TG. D LDL-C, E HDL-C, F ALT, G AST, H TNF- $\alpha$, I IL-6, J IL-2. K L The relative protein expression of GSK-3 $\beta$ and phosphorylation of INSR in liver using western blot analysis. Scale: $50 \mu \mathrm{m}$. The data are exprssed as mean $\pm \mathrm{SD} \quad * \mathrm{P}<0.05$ 


\section{A}
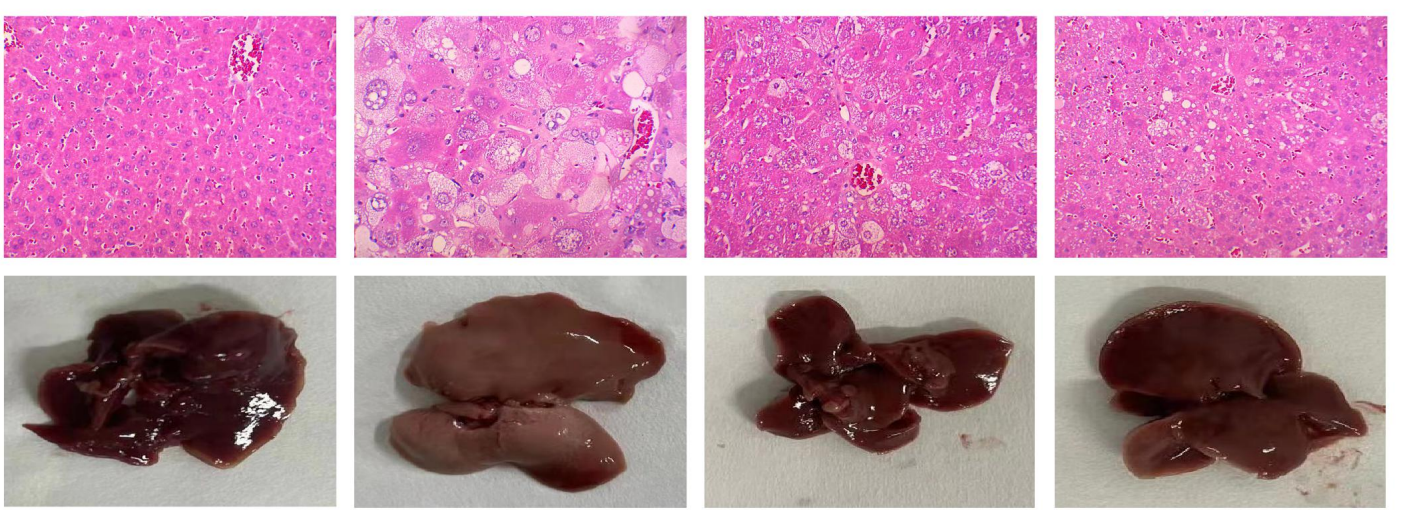

B

C

TG

D

LDL-C
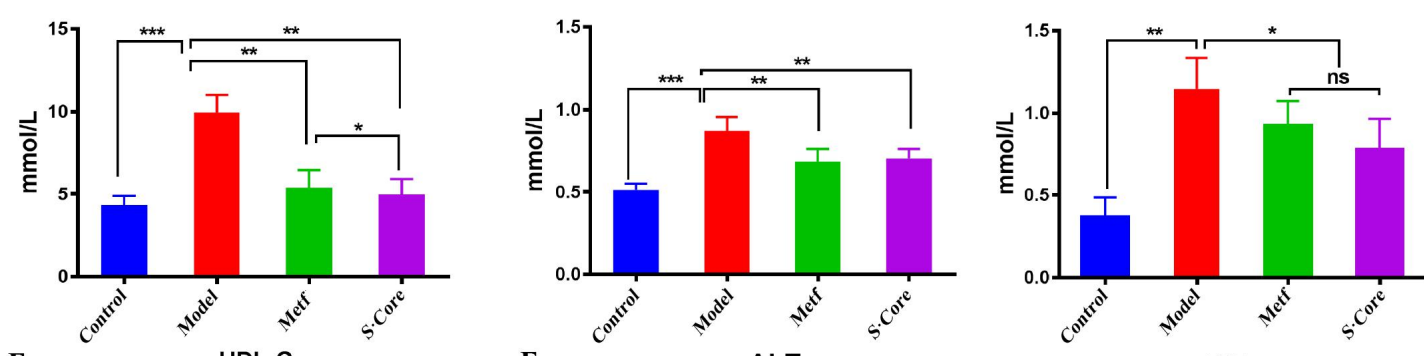

E

HDL-C

F

ALT
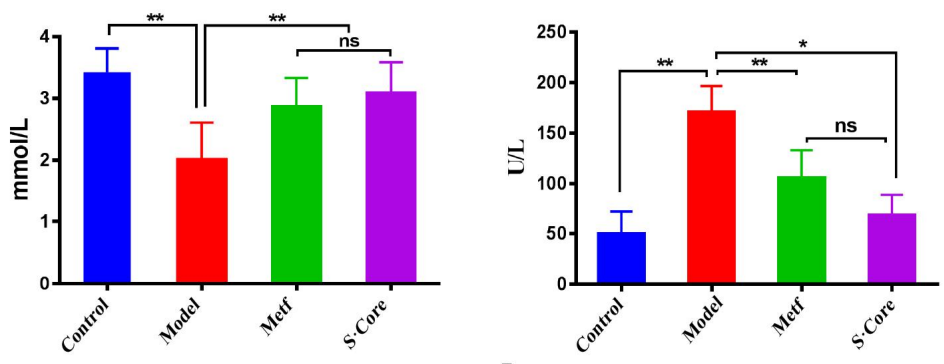

G

AST

H

TNF- $\alpha$

I

IL-6
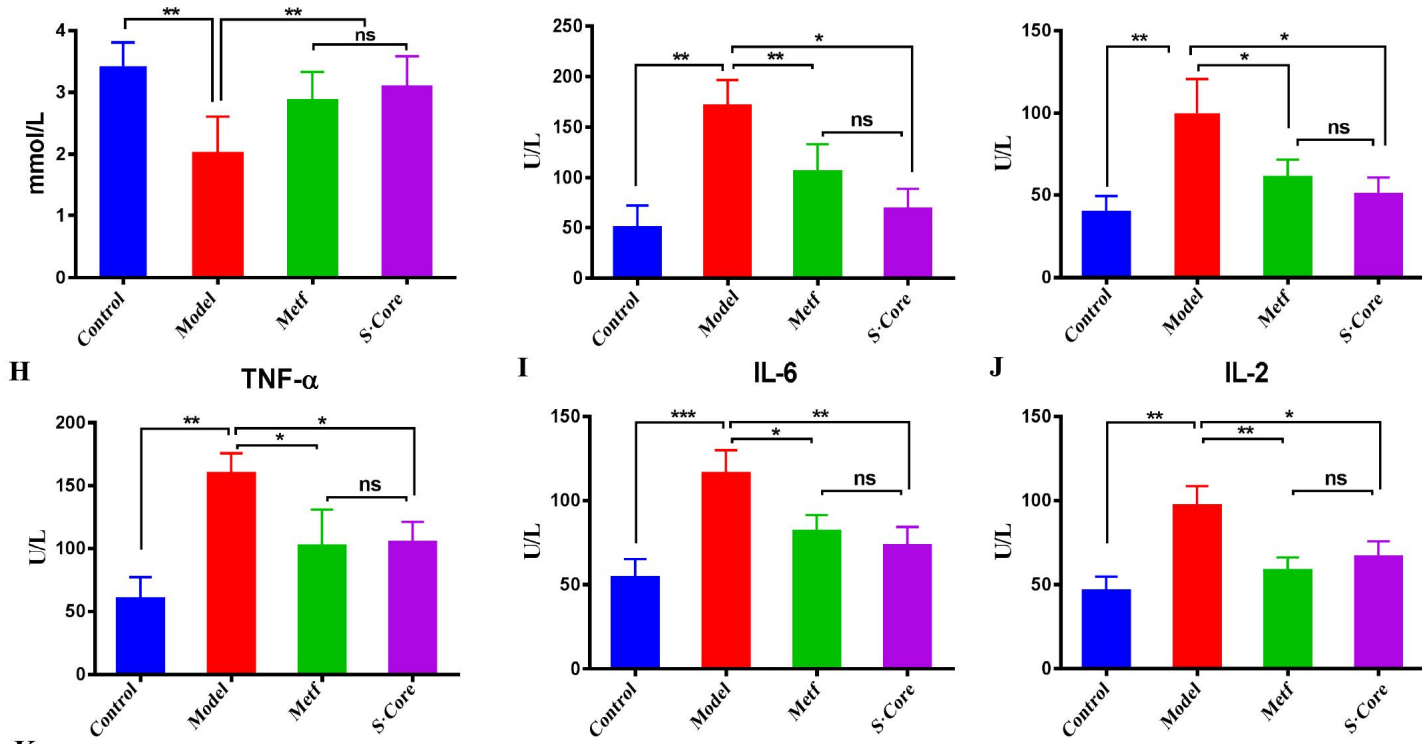

IL-2

K

L
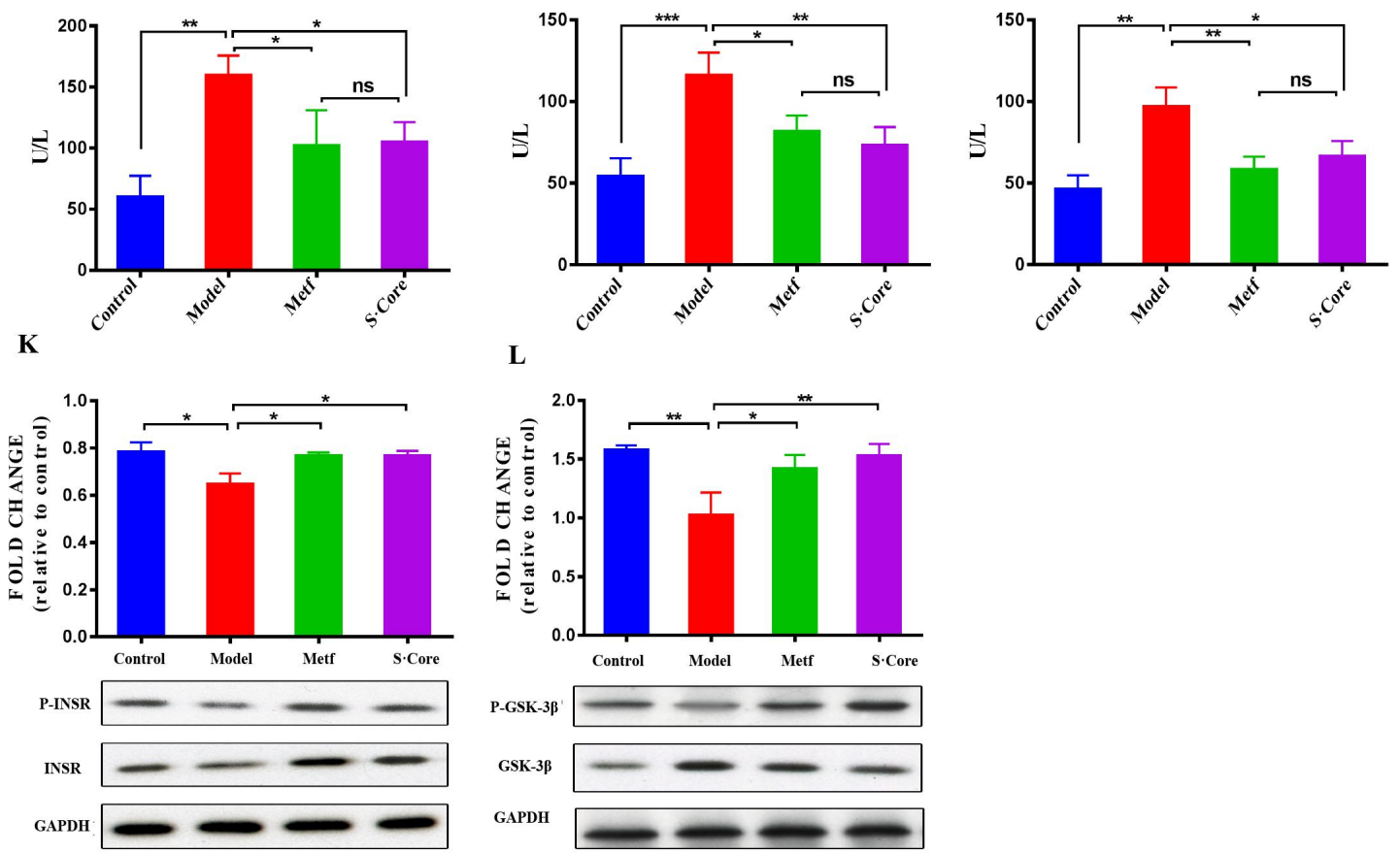


\section{Supplementary Files}

This is a list of supplementary files associated with this preprint. Click to download.

- renamed06793.xls

- renamed5b9f9.xlsx

- renamed8a4f3.xls

- renameda4c41.xIsx

- renamedf3f7f.xlsx 